
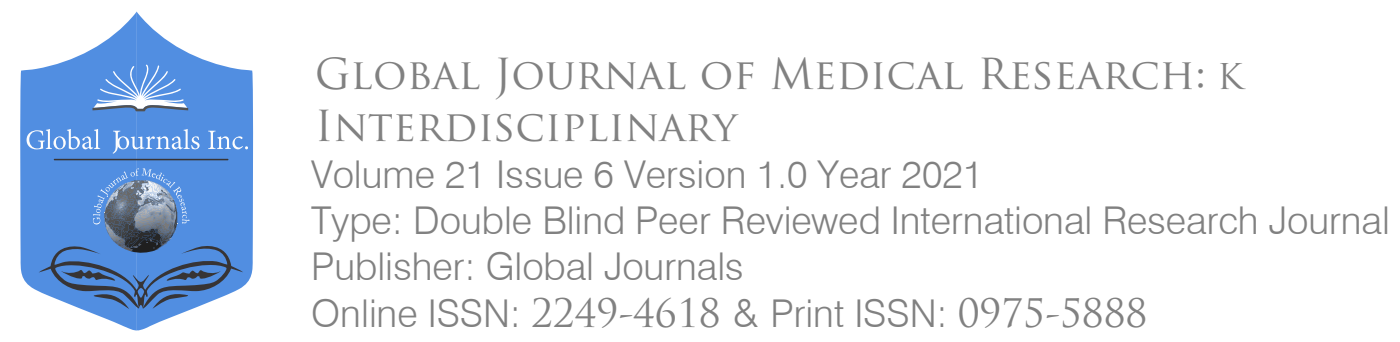

\title{
Postoperative Outcome Analyses of Non-Complicated Macula-Off Rhegmatogenous Retinal Detachment: A Retrospective, Long-Term, Multicenter Case Series Report
}

By Miguel A. Quiroz-Reyes, Erick A. Quiroz-Gonzalez, Jorge Morales-Navarro, Felipe Esparza-Correa, Jorge E. Aceves-Velazquez, Jennifer H Kim-Lee, Alejandra Nieto-Jordan, Margarita Montano, Virgilio Lima-Gomez

\& Federico Graue-Wiechers

Abstract- Background: There is abundant and even confusing information in the available literature concerning the role of internal limiting membrane (ILM) removal in macular conditions secondary to non-complicated macula-off rhegmatogenous retinal detachment (RRD) repair. This retrospective, multicenter, long-term study aimed to analyze the incidence of epiretinal membrane (ERM) proliferation and other surgical complications and to compare the postoperative microstructural and multimodal imaging findings and correlate them with the final postoperative best-corrected visual acuity (BCVA) in selected eyes.

Keywords: brilliant blue dye; epiretinal membrane; internal limiting membrane; macula-off retinal detachment; non-complicated rhegmatogenous retinal detachment; primary vitrectomy.

GJMR-K Classification: NLMC Code: WW 270

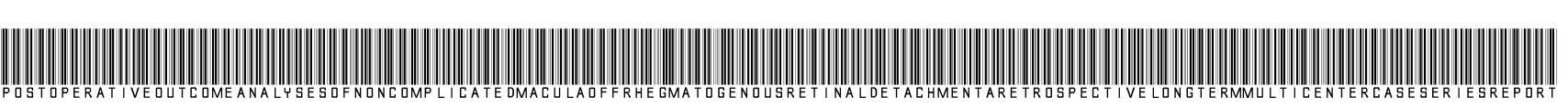

Strictly as per the compliance and regulations of:

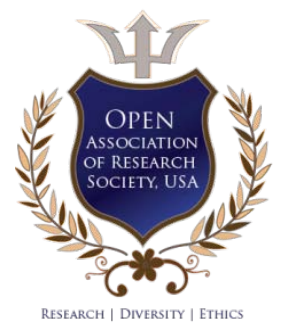

(c) 2021. Miguel A. Quiroz-Reyes, Erick A. Quiroz-Gonzalez, Jorge Morales-Navarro, Felipe Esparza-Correa, Jorge E. AcevesVelazquez, Jennifer H Kim-Lee, Alejandra Nieto-Jordan, Margarita Montano, Virgilio Lima-Gomez \& Federico Graue-Wiechers. This is a research/review paper, distributed under the terms of the Creative Commons Attribution-Noncommercial 3.0 Unported License http://creativecommons.org/licenses/by-nc/3.0/), permitting all non-commercial use, distribution, and reproduction in any medium, provided the original work is properly cited. 


\title{
Postoperative Outcome Analyses of Non- Complicated Macula-Off Rhegmatogenous Retinal Detachment: A Retrospective, Long- Term, Multicenter Case Series Report
}

\author{
Miguel A. Quiroz-Reyes ${ }^{\alpha}$, Erick A. Quiroz-Gonzalez ${ }^{\sigma}$, Jorge Morales-Navarro ${ }^{\rho}$, Felipe Esparza-Correa ${ }^{\omega}$, \\ Jorge E. Aceves-Velazquez ${ }^{*}$, Jennifer H Kim-Lee ${ }^{\S}$, Alejandra Nieto-Jordan ${ }^{x}$, Margarita Montano v , \\ Virgilio Lima-Gomez ${ }^{\ominus}$ \& Federico Graue-Wiechers ${ }^{\zeta}$
}

\begin{abstract}
Background: There is abundant and even confusing information in the available literature concerning the role of internal limiting membrane (ILM) removal in macular conditions secondary to non-complicated macula-off rhegmatogenous retinal detachment (RRD) repair. This retrospective, multicenter, long-term study aimed to analyze the incidence of epiretinal membrane (ERM) proliferation and other surgical complications and to compare the postoperative microstructural and multimodal imaging findings and correlate them with the final postoperative best-corrected visual acuity (BCVA) in selected eyes.

Methods: This long-term retrospective study included 230 eyes divided into three groups according to the surgical management performed for uncomplicated macula-off RRD: 125 eyes in the buckle group underwent scleral buckle techniques; 55 eyes in the non-peeling group underwent primary vitrectomy with no ILM peeling; 50 eyes in the peeling group with primary preoperative or secondary postoperative presence of significant ERM proliferations underwent the ERMILM en-bloc complex removal or double-staining removal techniques.
\end{abstract}

Results: The postoperative incidence of ERM was $23.2 \%$ (29 eyes) in the buckle group, $23.63 \%$ (13 eyes) in the non-peeling group, and $2.0 \%$ (one eye) in the peeling group $(p<0.05$; Student's t-test). The mean postoperative BCVA difference among the buckle group, peeling group, and non-peeling group was significant (logarithm of the minimum angle of resolution, $0.40 \pm 0.33$ vs. $0.47 \pm 0.16$ vs. $0.28 \pm 0.19$, respectively). Postoperative multimodal imaging tests yielded abnormal retinal thickness in the three groups, with a diffuse

Author $\sigma \rho \omega ¥ \S \chi \zeta$ : Institute of Ophthalmology, Conde de Valenciana, Private Assistance Institution (Non-profit organization), Chimalpopoca 14, Colonia Obrera, Mexico City 06800, Mexico.

Author a v: Oftalmologia Integral ABC and Retina Specialists SC at the American British Cowdray, Private Assistance Institution (Non-profit organization), Av. Carlos Graef Fernandez 154, Cuajimalpa de Morelos, Mexico City 05300, Mexico.

Author $\theta$ : Juarez Hospital, Public Assistance Institution (Non-profit organization), Av. Politecnico Nacional 5160, Colonia Magdalena de las Salinas, Mexico City 07760, Mexico.

Corresponding Author a: Institute of Ophthalmology, Fundacion Conde de Valenciana. Private Assistance Institution (IAP). Non-profit Organization. Mexico City, Mexico, Chimalpopoca 14 street, Colonia Obrera, Mexico City 06800, Mexico. e-mail: drquiroz@prodigy.net.mx optic nerve fiber layer and ellipsoid band disruptions predominantly in the peeling group, and a normal foveal profile in the buckle and non-peeling groups.

Conc/usions: Multiple structural alterations in spectral-domain optical coherence tomography biomarkers and a significant reduction in retinal sensitivity were observed in the peeling group. Eyes that developed secondary ERM proliferations in the buckle group and in the non-peeling group showed statistically significant upgrading in BCVA once the ERM proliferation and ILM were removed. Ultimately, our study contributes findings pertaining to severe consequences in macular structure and function. We can conclusively state that ILM removal with the main objective of avoiding macular ERM proliferation is not justified because of the high rate of potential macular complications and poor visual results.

Keywords: brilliant blue dye; epiretinal membrane; internal limiting membrane; macula-off retinal detachment; non-complicated rhegmatogenous retinal detachment; primary vitrectomy.

\section{BACKGROUND}

$\mathrm{M}$ ultiple surgical complications associated with scleral buckle surgery have been reported in the management of primary and non-complicated macula-off rhegmatogenous retinal detachment (RRD). Partial- or full-thickness scleral perforations can give rise to various serious trans-operative vitreoretinal complications, including retinal perforation with vitreoretinal entrapment, choroidal hemorrhage, and subretinal bleeding, that allow access to the submacular space with well-known deleterious effects on the photoreceptors. In addition to epiretinal membrane (ERM) proliferation after scleral perforation in buckle and cryotherapy surgery, the most commonly encountered postoperative complications are macular ectopia due to vitreomacular traction and proliferative vitreoretinopathy (PVR) with recurrent and complicated RRD[1-4].

According to the 2005-2019 trending data from the American Society of Retinal Specialists Preferences and Trends Survey [5], primary vitrectomy is the chosen procedure for non-complicated RRD cases not requiring a supplemental scleral buckle in order to reduce the 
aforementioned complications [5]. However, the incidence of macular complications, such as the appearance of epiretinal macular membranes, remains high. Several reports have shown that if the internal limiting membrane (ILM) is removed at the same time as the reapplication of the retina via primary vitrectomy and endolaser treatment, the incidence of significant ERM proliferations is reduced, and thus, additional surgical procedures can be avoided. However, ILM removal still has possible transoperative or postoperative structural and functional complications because the ILM acts as a scaffold for the proliferation of the glial and Muller cells; these cells create ERM proliferations that exert a tangential contraction over the macula $[6,7]$. Thus, the potential benefits of prophylactic ILM removal remain controversial [8-12].

The main objectives of this study were as follows: (1) to retrospectively determine the postoperative incidence of ERM proliferation over the macula and other postoperative surgical complications; (2) to analyze the long-term final postoperative structural, optical coherence tomography (OCT) findings; (3) to contribute to the analysis of macular microperimetry and multifocal electroretinography (mfERG) findings; and (4) to correlate these results with the final postoperative best-corrected visual acuity (BCVA) in different surgical management methods performed for uncomplicated macula-off RRD.

\section{Methods}

The Retina Department at the Institute of Ophthalmology. Oftalmologia Integral $\mathrm{ABC}$ and Retina Specialists at the American British Cowdray Hospital, and the Retina Service of the Hospital Juarez in Mexico City, Mexico, provided authorization and released the electronic clinical records for the database used in this study. This retrospective, long-term, multicenter, onesurgeon study adhered to the tenets of the Declaration of Helsinki, received full ethical approval from the Research Ethics Committees, and was approved by the Institutional Review Committees and the Teaching Departments of the three participating institutions (no reference number is provided for retrospective studies by these institutions). Written informed consent before the surgical procedure in accordance with the institutional guidelines was obtained from all the patients. Data are available from the Imagenology and Psychophysics Laboratory at the Retina Departments of the three institutions.

The study was designed to comparatively analyze the anatomical and functional outcomes of scleral buckle procedures and vitrectomy techniques with and without ILM removal, to evaluate the postoperative incidence of significant macular ERM proliferations and other main transoperative- and postoperative-related complications in 230 eyes of 164 patients from May 2014 to January 2021. The total sample population was divided into three groups according to the surgical management of noncomplicated macula-off RRD: buckle eye group, vitrectomy non-peeling eye group, and vitrectomy peeling eye group. Postoperative eyes that eventually developed significant secondary ERM proliferation over the macula and underwent a second surgical procedure for ERM removal were included in the peeling group. The postoperative redetachment rate was defined in the three surgical groups, and only eyes where the retina was successfully reattached for a minimum of 6 months of follow-up after the first or second surgical procedure were included in the general dataset. Thus, the final sample was composed of 230 eyes of 164 patients that met the inclusion criteria. The scleral buckle group included 125 eyes with no evidence of preoperative ERM proliferation and underwent $360^{\circ}$ scleral buckle surgery, rhegmatogenous lesions limited cryotherapy retinopexy, and additional subretinal fluid exo-drainage in selected cases. The non-peeling group included 55 eyes without evidence of preoperative ERM proliferation and underwent primary vitrectomy with no ILM removal. Ten eyes with a significant preoperative presence of primary ERM proliferation over the macula that underwent additional planned macular ERM-ILM complex (en-bloc removal), or double-staining technique removal were assigned to the peeling eye group. Owing to the long-term follow-up of these patients, the methodology of the study made it possible to add 27 eyes from the buckle group and 13 eyes from the non-peeling group that developed significant secondary macular ERM proliferation after the first procedure to the peeling eye group; all cases had at least 6 months of postoperative follow-up after the second surgical approach, consistent with vitrectomy and vitrectomy revision with ERM-ILM complex (en-bloc excision) or two-step (double-staining) removal techniques. To exactly differentiate the complications associated with a scleral buckle from those of vitrectomy with a complimentary buckle, all vitrectomy eyes on which a supplemental scleral buckle was placed were not included in this report.

Only the charts of patients aged 18 years or older who fulfilled the inclusion criteria of a noncomplicated macula-off RRD, non-myopia-related RRD (axial length $<26.5 \mathrm{~mm}$ ), no evidence of complicated $\mathrm{RRD}$, presence of primary ERM proliferation, presence of secondary ERM from the buckle and ILM peeling groups without recurrent RRD, at least 6 months of follow-up, and at least one well-documented structural and functional assessment of the macula at the last follow-up visit evaluation. The exclusion criteria were as follows: prior complicated vitreoretinal surgery or intravitreal injections, trauma-related RRD, occlusive vascular tractional detachment with a rhegmatogenous component, proliferative diabetic retinopathy-related 
combined rhegmatogenous and tractional $\mathrm{RD}$ or macular diabetic tractional RD, RRD associated with a giant retinal tear, myopic traction maculopathy macular hole associated to RRD, severe PVR recurrent and complicated RRD, presence of intravitreal silicone oil, history of active glaucoma, and placement of a supplemental scleral buckle. The elimination criteria were an impossibility for follow-up, loss of follow-up, surgery in a non-designated institution, presence of severe complications such as endophthalmitis, recurrent, complicated RRD at the last follow-up visit evaluation, and refractory corneal opacity development during follow-up.

The following postoperative assessments were statistically analyzed for the eyes in the three groups (buckle, non-peeling, and peeling groups): Long-term postoperative structural spectral-domain optical coherence tomography (SD-OCT) findings including central subfoveal thickness (CSFT), foveal contour, central subfoveal ellipsoid band status, ELM line appearance, en-face imaging analysis for the presence of dissociated optic nerve fiber layer (DONFL) defects, and the presence of ERM proliferation over the macula. Postoperative multimodal functional evaluations included the final BCVA in logarithm of the minimum angle of resolution (logMAR) units, macular retinal sensitivity (MRS), foveal retinal sensitivity (FRS), and retinal sensitivity analysis mapping assessed by microperimetry with the standard Macular Integrity Assessment (MAIA) examination standard protocol covering a $10^{\circ}$ diameter area with 37 measurements points and a light stimulus projected directly over the macula surface, with a size stimuli of Goldman III, background luminance of 4 apostilbs (asb) and maximum luminance of $1000 \mathrm{asb}$, and 36 decibels (dB) dynamic range. Fixation stability and fixation location patterns parameters are assessed by tracking eye movements 25 times/second and by plotting the resulting distribution over the scanning laser ophthalmoscope image, each movement is represented by a point, and the overall site describes the preferred retina locus (PRL). Computerized mfERG was used to detect focal (regional) outer retinal abnormalities, the amplitude and implicit time of the N1 wave, implicit time of the P1 wave, and elevation electroretinography 3-D maps were assessed in the affected eye and compared to the normal contralateral eye or to the corresponding control normative dataset. 61-hexagon $30^{\circ}$ standardized technique to test the macular electrical multifocal outer layers sensitivity point to point at the $<2$-degree to $>15$ degree central rings $(<2,2-5,5-10,10-15,>15$ central rings) was performed at the last follow-up evaluation visit.

\section{a) Examinations}

A total of 230 eyes of 164 patients underwent a general ophthalmic evaluation and preoperative examinations, including BCVA assessment, biomicroscopy slit-lamp examination, fundus examination through a panfundoscopic contact lens, and indirect ophthalmoscopy. Cross-sectional images of the macular region were acquired along the horizontal plane through the foveal center using SD-OCT (RTVueXR platform SD-OCT, Optovue, Inc., Fremont, CA, USA), and the axial lengths were measured using partial coherence laser interferometry (Zeiss IOL Master 700; Carl Zeiss Meditec AG, Oberkochen, Germany). The presence of a simple, non-complicated macula-off RRD or non-complicated, recurrent macula-off RRD in the three groups was confirmed by indirect ophthalmoscopy and B-scan ultrasonography (A and B Ultrasound Unit, Quantel Medical, Du Bois Loli, Auvergne, France). The postoperative microstructural evaluation was performed using SD-OCT (Spectralis OCT Heidelberg Engineering. Heidelberg, Germany) and a swept-source (SS)OCTdevice (Topcon Medical Systems, Inc., Oakland, NJ, USA) in some cases, while postoperative functional macular evaluation was conducted with microperimetry (MP-3 MAIA Confocal Microperimeter by Metrovision, Pérenchies, France) and mfERG testing (Electrophysiology Vision Monitor Analyzer, Model MonPackONE by Metrovision). All OCT images, mfERG and microperimetry testing were analyzed by three experienced retinal co-authors from the three participating institutions.

\section{b) Surgical procedures}

A methodical, standardized, classical scleral buckle surgical procedure was performed (by one of the authors MAQR) in the buckle group consistent with traditional 505,504 , or $503,360^{\circ}$ round Lincoff episcleral sponges (Storz model E-5395-4) and oval foam silicon sponges (506 style S 1981-5 or 501 style S 1981-4) with the newly designed profile (Labtician Ophthalmics, Inc., Ontario, Canada) around the equator of the eye and fixed with polyester 5-0 MERSILENE ${ }^{\circledR}$ Polyester Sutures, double-armed $3 / 8$ circle spatulated needle suture (ETHICON, Johnson \& Johnson, Brunswick, NJ, USA). According to the morphological appearance of the RRD, transscleral subretinal fluid (SRF) drainage assisted with a 7-0 vicryl polyglactin suture (needle P-1, 3/8 c, reverse cutting; ETHICON) was performed through the scleral wall on the selected meridian site based on previous visualization and location of large choroidal vessels to avoid potential subretinal or choroidal bleeding, which was prevented or treated by diathermy if necessary, after the SRF drainage. The eye volume and pressure lost were recovered with sterilized air. Only in the buckle group, before or after the retina was reattached, limited transscleral cryotherapy over or around the suspected rhegmatogenous lesions, preferably after retina reattachment to avoid retinal pigment epithelium (RPE) cell dispersion was applied with the assistance of a binocular indirect ophthalmoscope and a 20-diopter 
condenser lens. The tenon capsule and conjunctival tissue were repositioned, carefully sutured, and fixed to the episcleral tissue with the same 7-0 vicryl polyglactin to protect the exoplant and prevent infections, conjunctival erosions, and exoplant extrusions. In the vitrectomy groups, a standard 23- or 25-gauge threeport pars plana vitrectomy (Alcon Constellation Vision System. Alcon Labs, Fort Worth, TX, USA) with a total vitreous release of the retina was performed in all eyes under local anesthesia plus sedation by one of the authors (MAQR). The vitrectomy was performed using a contact wide-angle viewing precorneal lens system (ROLS reinverted system Volk Medilex, Miami, FL, USA), the Wide Angle Viewing System with non-contact lens (Insight Instruments, Inc. Stuart, FI. USA), or recently in the last seven cases, the Zeiss ARTEVO 800 digital ophthalmic 3-D head-up microscope with the Resight non-contact lens system (Carl Zeiss Meditec AG, Jena, Germany); this new digital microscope with a hybrid mode (coaxial and 3-d HD 4K monitor) and integrated transoperative OCT allowed for real-time retinal structural analysis and detection of ERM proliferation, thus enabling a more precise membrane stripping (Figure1D to D-5). In addition to central vitrectomy, our standard technique used a diluted triamcinolone acetonide adjuvant (Kenalog $40 \mathrm{mg} / \mathrm{mL}$; Bristol-Myers Squibb, New York, NY, USA) to identify and better visualize the vitreous and its base and to safely perform integral removal of its cortical face from the surface of the retina using a silicone-tipped cannula with active suction prior to perfluorocarbon liquid (PFCL) infusion and reattachment of the retina, focusing on achieving a free and mobile posterior hyaloid face. The retina was reattached by a PFCL-assisted technique to effectively perform hydropneumatic retinal manipulation and assisted SRF endodrainage in all the vitrectomy eyes (peeling and non-peeling groups).

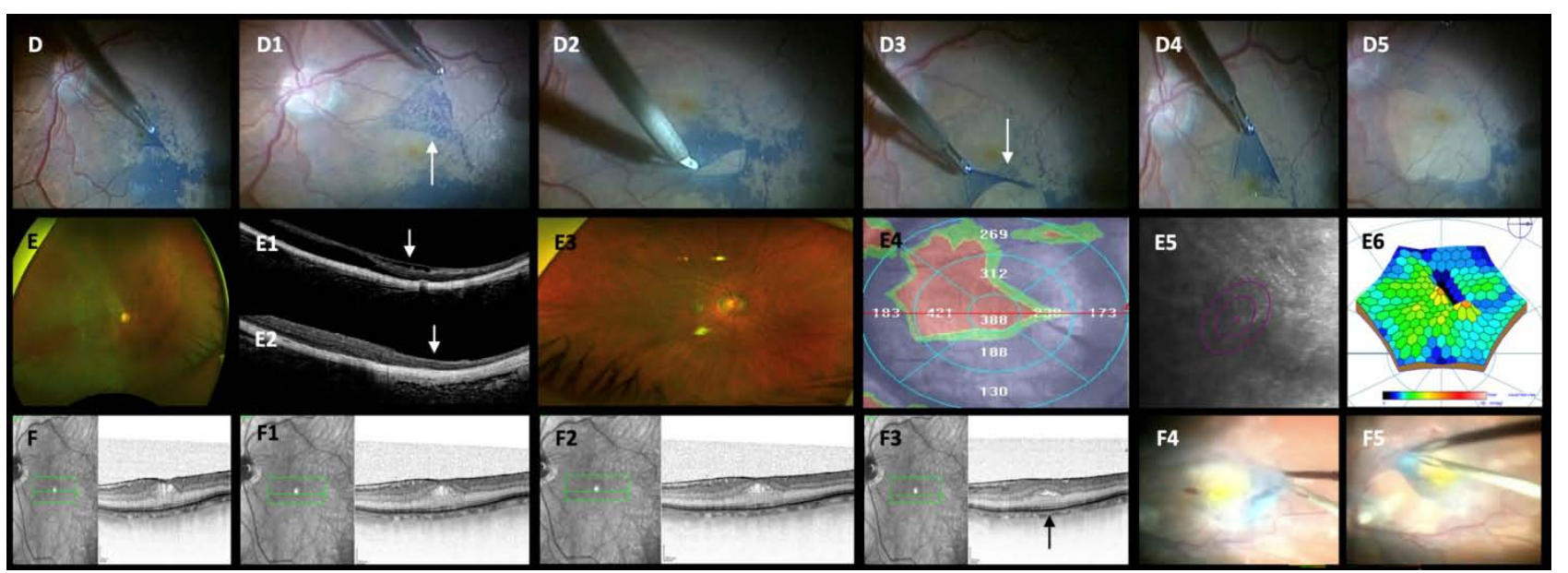

Figure 1: Postoperative structural and functional findings (part 2). (D)-(D-5) Sequence of epiretinal membrane (ERM)-internal limiting membrane (ILM)two-step removal technique events. (D) and (D-1) First-step removal of the dyed trypan blue ERM proliferation (white arrow). (D-2)-(D-5) Uncomplicated second step Brilliant Blue G-dyed ILM removal (white arrow); in this case (case 67), the final best-corrected visual acuity (BCVA) is 0.18 logarithm of the minimum angle of resolution (logMAR). (E) An Optos photo showing rhegmatogenous retinal detachment with macular wrinkling due to postoperative ERM proliferation; the extramacular retina is attached. (E-1) Spectral-domain optical coherence tomography (SD-OCT) of macular thickening with loss of foveal contour due to ERM proliferation and residual subretinal fluid (FRS) (white arrow). (E-2) Corresponding retinal thickening and retinal thinning 6 months after ERM-ILM removal; the ERM, ELM, and ellipsoid band cannot be demonstrated. (E3) AnOptos, colorcorresponding photo.(E-4) Abnormal topographic thickness retinal map with irregular and diffuse macular thickening at the end of the follow-up. (E-5) Postoperative microperimetry depicting eccentric and unstable fixation patterns; macular retinal sensitivity and FRS are abnormal, and the retinal sensitivity analysis map shows abnormal macular integrity. (E-6) Abnormal three-dimension topographic map of the macular area showing a very abnormal response due to foveal photoreceptors and bipolar cell sensitivity deep reduction, with very abnormal spatial resolution due to unstable fixation and location (locus) patterns. The final postoperative BCVA after ERM removal, in this case,is0.70 logMAR.(F)-(F-3) Spectralis SD-OCT scans through the center of the macula depicting ERM proliferation associated with diffuse retinal thickening and retinal superficial layer wrinkling with cystic spaces in the Henle nerve fibers; the ELM line shows some attenuation, and the ellipsoid band is disrupted (black arrow). (F-4) and (F-5) Macular surgery sequence of the two-step technique of dyed ERM-ILM complex removal (case 66) 8 weeks after ERM-ILM removal, BVCA is 0.60 logMAR.

The vitreous base was shaved $360^{\circ}$, assisted with scleral depression in all the eyes that underwent

vitrectomy; this scleral depression allowed removal of the vitreous traction completely from flap tears and 
careful shaving and debulking of the vitreous base using mostly closed port duty cycle and low infusion pressure, even in areas of a detached retina, without producing iatrogenic retinal tears. Our young patients generally showed vitreous that was attached or only partially detached, and removal of the core vitreous was relatively straightforward; however, separation of the posterior hyaloid and other areas of adherent vitreous in the periphery with a very mobile retina was technically intricate, especially when concurrent lattice degeneration was present. Once the retina was reattached and in the absence of a scleral buckle, performing meticulous peripheral vitrectomy and ensuring that all retinal tears were identified and laser treated, were crucial; a benefit of vitrectomy in these groups was that it allowed for the removal of all vitreous opacities, treated the opacified lens capsules, and addressed the cases where macular ERM proliferation was pre- or trans-operatively confirmed. Surgical macular staining was performed using $0.15 \mathrm{~mL}$ of a 0.25 $\mathrm{mg} / \mathrm{mL}(0.025 \%)$ diluted isomolar solution $(\mathrm{pH} 7.4)$ of Brilliant Blue $G$ dye (BBG), to selectively stain and peel off the ILM along with the ERM (en-bloc removal technique). For the ILM-ERM en-bloc removal technique (Figure1 F-4, F-5), a 23- or 25-gauge diamond-dusted membrane scraper and 25-gauge 0.44 ILM forceps (Grieshaber Revolution DSP ILM forceps; Alcon Labs, Fort Worth, TX, USA) and a 23- or 25-gauge Finesse ILM flex loop microinstrument (Grieshaber; Alcon Labs) to facilitate the ERM and ILM removal from arcade to arcade were used. In cases where the removal was performed in two steps (double staining technique), first, trypan blue $0.15 \%$ ophthalmic solution (Membrane Blue; Dutch Ophthalmic, USA) was instilled under air to remove the ERM proliferations after washing the dye; afterwards, the MLI was stained with the aforementioned BBG dye, followed by removal (en-bloc or double staining technique removal).

We performed SRF endodrainage by creating a tiny site-selected drainage retinotomy or using preexisting endodiathermy-marked retinal breaks. First, fluid to fluid exchange was done over the retinal break to remove viscous protein aceous SRF, and also to reduce the extent of SRF and minimize the chance of trapped SRF before proceeding to an air-fluid exchange and continuing with SRF drainage. Once the retina was completely free of vitreous traction and completely reattached, argon laser endophotocoagulation around the rhegmatogenous lesions and suspected retina areas was thoroughly performed; to completely dry out the subretinal space, a second air-fluid exchange was performed, and as the last surgical step, a nonexpandable bubble containing 15\% perfluoropropane (C3F8) gas mixture was used as a long-acting tamponade at the end of the procedure in all the cases.

\section{c) Statistical analyses methodology}

A post-hoc power test was used to determine the power of the analyses, and descriptive and analytic statistics were employed to analize our data. Variability of the numerical variables was measured and reported as mean \pm standard deviation (SD). The categorical variables are reported as counts (\% frequency). For the statistical analyses, all Snellen visual acuities were converted to logMAR visual acuities according to the following formula: logMAR = -log (decimal acuity).

To determine the statistical test required, the Shapiro-Wilk normality test was used to investigate if the variables followed a normal distribution; per the results, the non-parametric Mann-Whitney U-test was used to investigate the associations of the preoperative BCVA, postoperative BCVA, and final BCVA after ERM proliferation removal in terms of the differences in medians with the numerical variables. The Kruskal-Wallis test was used to examine potential differences of the preoperative BCVA, postoperative BCVA, and final BCVA after ERM proliferation removal among the categorical variables. Furthermore, the Wilcoxon ranksum test was used for the numeric variables, and Fisher's exact test for the categorical variables listed to investigate if the variables presented showed significant differences among the buckle, non-peeling, and peeling eye groups. Spearman's rank correlation coefficient (rho) tests investigated the potential correlations among the numeric variables listed. A generalized linear model (GLM) further investigated potential associations of the preoperative BCVA, postoperative BCVA, and final BCVA after ERM proliferation removal with the other variables listed. To determine the best model for each of these variables, a stepwise algorithm was used to choose the Akaike information criterion (AIC) model from the package step [13]. We set the significance of our tests to be $p<0.05$. For all statistical analyses, we used R (R Foundation for Statistical Computing, Vienna, Austria; https://www.R-project.org/). Additionally, the collected data were statistically analyzed using IBM SPSS for Windows, version 25.0 (IBM Corp. Armonk, NY. USA). The BCVA was evaluated with the Student's t-test for related samples (statistical hypothesis test in which the test statistic follows Student's t-distribution under the null hypothesis and is used to determine if the means of two sets of data are significantly different from each other); a result of $p<0.05$ was considered statistically significant.

\section{Results}

\section{a) Results in the Buckle group}

The power of the analysis was very good (Power $=99.9 \%)$ for the given sample size $(n=125)$ and for a medium effect size (Cohen's $d=0.5$ ). The results of the Shapiro-Wilk normality test showed that most of the numerical data followed a normal distribution $(p<0.05)$; 
hence, we decided to use the non-parametric MannWhitney U-test to investigate the associations of the preoperative BCVA, postoperative BCVA, and final BCVA after ERM proliferation removal, in terms of the differences in medians of these variables (Additional Tables S1, S2).

We examined 125 eyes in the buckle group, comprising 59 (47.2\%) left eyes and 66 (52.8\%) right eyes. From these eyes, 98 (78.4\%) were in the phakic group, and 27 (21.6\%) were in the pseudophakic group; the state of the lens was not statistically analyzed. The mean age of the study population was $44.3( \pm 15.9)$ years, of which 75 (60.0\%) were females, and 50 $(40.0 \%)$ were males. The mean preoperative period with the macula-off before surgery was $3.6( \pm 2.5)$ weeks and the mean postoperative follow-up period was 26.1 ( \pm 13.4$)$ months with 31 eyes (24.8\%) with 20/40 visual acuity or better at the end of follow-up (Table 1 and Additional Table S2).

Table 1: Baseline characteristics of the three groups

\begin{tabular}{lcccc}
\hline \multicolumn{1}{c}{ Variable } & $\begin{array}{c}\text { Buckle } \\
\text { group } \\
(\mathrm{N}=125)\end{array}$ & $\begin{array}{c}\text { Non-peeling group } \\
(\mathrm{N}=55)\end{array}$ & $\begin{array}{c}\text { Peeling } \\
\text { group } \\
(\mathbf{N}=50)\end{array}$ & $\begin{array}{c}\text { P-value } \\
\text { significance }\end{array}$ \\
\hline $\begin{array}{l}\text { Age (mean) } \\
\text { Sex }\end{array}$ & $44.3 \pm 15.9 \mathrm{sd}$ & $50.4 \pm 13.5 \mathrm{sd}$ & $45.12 \pm 15.3 \mathrm{sd}$ & 0.054 \\
-Female & $75(60 \%)$ & $19(34.5 \%)$ & $18(36 \%)$ & 1.00 \\
-Male & $50(40 \%)$ & $36(65.5 \%)$ & $32(64 \%)$ & 0.068 \\
$\begin{array}{l}\text { Preop lens status } \\
\text {-Phakic }\end{array}$ & $48(78.4 \%)$ & $31(36.4 \%)$ & $37(74 \%)$ & 0.425 \\
-Pseudophakic & $27(21.6 \%)$ & $24(43.6 \%)$ & $13(26 \%)$ & 0.131 \\
$\begin{array}{l}\text { Preop macula-off } \\
\text { (weeks) }\end{array}$ & $3.6 \pm 2.5 \mathrm{sd}$ & $4.52 \pm 2.4 \mathrm{sd}$ & $4.30 \pm 2.7 \mathrm{sd}$ & 0.386 \\
$\begin{array}{l}\text { Postop follow up } \\
\text { (months) }\end{array}$ & $26.12 \pm 13.4 \mathrm{sd}$ & $25.62 \pm 12.4 \mathrm{sd}$ & $22.66 \pm 13.54 \mathrm{sd}$ & $1.077 \pm 0.277 \mathrm{sd}$ \\
$\begin{array}{l}\text { Preop BCVA } \\
\text { (mean) }\end{array}$ & $1.03 \pm 0.28 \mathrm{sd}$ & $1.036 \pm 0.258 \mathrm{sd}$ & & \\
\hline
\end{tabular}

Preop, preoperative; Postop, postoperative; BCVA, best-corrected visual acuity; sd, standard deviation

Complete descriptive statistics for the numerical and categorical variables are presented in Table 2 and Additional Tables S2 and S3. The Spearman's rank correlation coefficient test showed that there was a moderate to strong positive correlation $(r h o=0.57$, $p<0.01$ ) of the postoperative BCVA in logMAR units with the BCVA after ERM surgery. In addition, there was a weak negative correlation ( $r$ o $=-0.2, p<0.05$ ) between postoperative BCVA in logMAR units and follow-up period in months (Additional Table S4; Additional Figure S1).

Table 2: Summary of postoperative outcomes in the three groups

\begin{tabular}{|c|c|c|c|c|}
\hline Variable & $\begin{array}{l}\text { Buckle } \\
\text { group } \\
(\mathrm{N}=125) \\
\end{array}$ & $\begin{array}{l}\text { Non-peeling group } \\
\qquad(\mathrm{N}=55)\end{array}$ & $\begin{array}{c}\text { Peeling } \\
\text { group } \\
(\mathrm{N}=50) \\
\end{array}$ & $\begin{array}{c}\text { P-value } \\
\text { significance }\end{array}$ \\
\hline Mean preop BCVA & $1.03 \pm 0.2 \mathrm{sd}$ & $1.036 \pm 0.25 \mathrm{sd}$ & $1.077 \pm 0.27 \mathrm{sd}$ & 0.386 \\
\hline Mean postop BCVA & $0.40 \pm 0.33 \mathrm{sd}$ & $0.28 \pm 0.19 \mathrm{sd}$ & $0.47 \pm 0.16 \mathrm{sd}$ & $<0.05$ \\
\hline ERM detection (weeks) & $11.93 \pm 4.54 \mathrm{sd}$ & $18.00 \pm 6.45 \mathrm{sd}$ & $12.57 \pm 4.38 \mathrm{sd}$ & 0.009 \\
\hline RRD recurrence rate & $8.8 \%$ & $1(1.82 \%)$ & $12(24 \%)$ & 0.001 \\
\hline $\begin{array}{l}\text { Mean BCVA before } \\
\text { ERM-ILM removal }\end{array}$ & $0.40 \pm 0.10 \mathrm{sd}$ & $0.297 \pm 0.23 \mathrm{sd}$ & $0.756 \pm 0.32 \mathrm{sd}$ & 0.001 \\
\hline $\begin{array}{l}\text { Mean final BCVA after } \\
\text { ERM-ILM removal }\end{array}$ & $0.43 \pm 0.14 \mathrm{sd}$ & $0.28 \pm 0.19 \mathrm{sd}$ & $0.48 \pm 0.16 \mathrm{sd}$ & $<0.05$ \\
\hline $\begin{array}{l}\text { Foveal contour } \\
\text { abnormalities }\end{array}$ & 19 eyes $(15.2 \%)$ & Six eyes (11.3\%) & 18 eyes (37.5\%) & $<0.05$ \\
\hline Mean CSFT (microns) & $243.57 \pm 41.95$ & $266.71 \pm 32.75 \mathrm{sd}$ & $253.073 \pm 35.66 \mathrm{sd}$ & 0.173 \\
\hline DONFL defects present & 31 eyes (24.8\%) & Five eyes (11.36\%) & 29 eyes (58\%) & $<0.05$ \\
\hline $\begin{array}{l}\text { IS/OS (ellipsoid band) } \\
\text { integrity }\end{array}$ & $\begin{array}{c}\text { Disrupted }=25 \text { eyes } \\
(20 \%) \\
\text { Normal }=86 \text { eyes } \\
(68.8 \%)\end{array}$ & $\begin{array}{c}\text { Disrupted }=16 \text { eyes } \\
(29.09 \%) \\
\text { Normal }=39 \text { eyes } \\
(70.40 \%)\end{array}$ & $\begin{array}{c}\text { Disrupted }=13 \text { eyes } \\
(26 \%) \\
\text { Normal }=37 \text { eyes }(74 \%)\end{array}$ & 0.002 \\
\hline
\end{tabular}




\begin{tabular}{|c|c|c|c|c|}
\hline ELM line appearance & $\begin{array}{c}\text { Abnormal }=24 \text { eyes } \\
(19.2 \%) \\
\text { Normal }=86 \text { eyes } \\
(68.8 \%)\end{array}$ & $\begin{array}{c}\text { Disrupted }=16 \text { eyes } \\
(29.09 \%)\end{array}$ & $\begin{array}{c}\text { Disrupted }=35 \text { eyes } \\
(76 \%)\end{array}$ & 0.654 \\
\hline mfERG alterations & $\begin{array}{c}\text { Abnormal }=54 \text { eyes } \\
(43.2 \%) \\
\text { Normal }=45 \text { eyes } \\
(36 \%)\end{array}$ & $\begin{array}{c}\text { Disrupted }=13 \text { eyes } \\
(33.3 \%)\end{array}$ & $\begin{array}{c}\text { Disrupted }=30 \text { eyes } \\
(88 \%)\end{array}$ & $<0.05$ \\
\hline $\begin{array}{l}\text { Microperimetry } \\
\text { alterations }\end{array}$ & $\begin{array}{c}\text { Abnormal }=51 \text { eyes } \\
(40.8 \%) \\
\text { Normal }=56 \text { eyes } \\
(44.8 \%)\end{array}$ & $\begin{array}{c}\text { Disrupted }=11 \text { eyes } \\
(25.6 \%)\end{array}$ & $\begin{array}{c}\text { Disrupted }=24 \text { eyes } \\
(70.6 \%)\end{array}$ & $<0.05$ \\
\hline $\begin{array}{l}\text { Follow-up period } \\
\text { (months) }\end{array}$ & $26.11 \pm 13.42 \mathrm{sd}$ & $24.80 \pm 12.34 \mathrm{sd}$ & $21.88 \pm 13.32 \mathrm{sd}$ & 0.133 \\
\hline
\end{tabular}

Preop, preoperative; BCVA, best-corrected visual acuity; ERM, epiretinal membrane; RRD, rhegmatogenous retinal detachment; ILM, internal limiting membrane; CSFT, central subfoveal thickness; IS/OS, internal segment/ external segment; DONFL, diffuse optic fiber layer; mfERG, multifocal electroretinogram; sd, standard deviation

The Mann-Whitney $U$ test showed that the preoperative BCVA in IogMAR units was statistically significantly different $(p<0.05)$ for the numeric variables such as age, preoperative period with the macula-off in weeks, postoperative BCVA in logMAR units, postoperative ERM detection in weeks, BCVA in logMAR units after ERM surgery, CSFT alterations (microns), and follow-up period in months (Additional Table S5 A). The postoperative BCVA in logMAR units was statistically significantly different $(p<0.05)$ for the numeric variables age, preoperative period with the macula-off in weeks, preoperative BCVA in logMAR units, postoperative ERM detection in weeks, BCVA in logMAR units after ERM surgery, CSFT alterations (microns), and follow-up period in months (Additional Table S5 B). Additionally, the BCVA in logMAR units after ERM surgery was statistically significantly different $(p<0.05)$ for the numeric variables age, preoperative period with the macula-off in weeks, preoperative BCVA in logMAR units, postoperative BCVA in logMAR units, postoperative ERM detection in weeks, CSFT alterations (microns), and follow-up period in months (Additional Table S5 C).

The Kruskal-Wallis test results showed that the preoperative BCVA in logMAR units was not statistically significantly different $(p>0.05)$ in the buckle group when correlated with any of the categorical variables; in other words, no correlation was found among the preoperative BCVA with any of the categorical variables (Additional Table S6 A). In addition, the postoperative BCVA in logMAR units was statistically significantly different $(p<0.05)$ among the following variables: redetachment, postoperative ERM proliferation, ERM proliferation surgery, BCVA after ERM proliferation surgery, presence of submacular blood, presence of alteration on SD-OCT, mfERG and microperimetry alterations (Additional Table S6 B). Furthermore, the BCVA in logMAR units after ERM surgery was not statistically significantly different $(p>0.05)$ among the groups of categorical variables (Additional Table S6 C).

The GLM for the postoperative BCVA in logMAR units showed that the postoperative BCVA in logMAR units was significantly dependent on the postoperative ERM proliferation, increasing the postoperative BCVA by 0.68 in $\log M A R$ units, and on retinal entrapment, reducing the postoperative BCVA by 0.21 in IogMAR units when adjusting for potential cofounders within the multivariable analyses (Additional Table S7). The GLM also showed that the final postoperative BCVA in logMAR units after ERM surgery was significantly dependent on the postoperative BCVA, preoperative BCVA in logMAR units, and retinal perforation, increasing the postoperative BCVA in logMAR units after ERM surgery by 0.15 logMAR units.

Summarizing the clinically important statistical findings in the buckle group, the Kruskal-Wallis test revealed that the preoperative BCVA, postoperative BCVA, and final BCVA after ERM surgery were compared with all the available variables. For the preoperative BCVA, we did not find any variable that was associated. The postoperative BCVA was statistically significantly associated $(p<0.05)$ with the following variables: the presence of a significant postoperative ERM proliferation, retina redetachment, ERM surgery, the presence of submacular blood, and the event of ERM proliferation removal surgery. For the final postoperative BCVA after ERM proliferation removal, we did not find any variables that showed a significant association. The GLM showed that the postoperative BCVA was statistically significant depending on the variables of postoperative ERM proliferation, increasing the postoperative BCVA by 0.68 in logMAR units, and retinal entrapment, reducing the postoperative BCVA by 0.21 in logMAR units. The GLM showed that the final postoperative BCVA after ERM proliferation surgery was statistically significant 
depending on the variables of postoperative BCVA logMAR units, preoperative BCVA in log MAR units, and retinal perforation, increasing the postoperative BCVA after ERM surgery by 0.15 in logMAR units.

\section{b) Results in the Vitrectomy groups}

The power of analysis for the vitrectomy groups (peeling and non-peeling groups) was very good (Power $=95 \%)$ for the given sample size $(n=105)$ and for a medium effect size $(d=0.5)$. The Shapiro-Wilk normality test(Additional Table S8)showed that none of the variables followed a normal distribution $(p<0.05)$; hence, we used the non-parametric Mann-Whitney Utest to investigate the associations of the preoperative BCVA, postoperative BCVA, and final BCVA after ERM proliferation removal, in terms of the differences in medians of these variables.

We examined 105 eyes in the vitrectomy groups, of which 50 (47.6\%) were left eyes and 55 (52.4\%) right eyes. The mean age of the study population was $48.9( \pm 14.6)$ years, of which $37(35.2 \%)$ were females and 68 (64.8\%) were males. The mean preoperative period with the macula-off before surgery was $4.4( \pm 2.6)$ weeks and the mean postoperative follow-up period was 23.4 ( \pm 12.9$)$ months (Table 1, Additional Table S9).

There were 50 eyes (47.62\%) in the peeling group, 27 eyes $(23.2 \%$ incidence of secondary ERM after buckle procedure) from the buckle group, 13 eyes (23.63\% incidence of postoperative secondary ERM proliferation after primary vitrectomy) from the nonpeeling group, and 10 eyes (4.34\% prevalence of ERM in primary non-complicated macula-off RRD in the whole sample studied in this report) initially diagnosed as having a primary ERM proliferation. The non-peeling group comprised 55 eyes (52.38\%).

The Wilcoxon rank-sum tests for the numeric variables (Additional Table S10) and the Fisher's Exact tests for the categorical variables showed that the variables such as first surgery (Additional Table S11), BCVA in log MAR units before ERM-ILM removal, recurrent RRD, additional surgery, postoperative ERM proliferation detection in weeks, final postoperative BCVA, postoperative foveal contour, presence of DONFL defects, mfERG and microperimetry alterations demonstrated statistically significant differences $(p<0.05)$ among the peeling and non-peeling groups (Table 2).

The Spearman's rank correlation coefficient test showed a strong positive correlation $(\mathrm{rho}=0.78$, $p<0.01$ ) of the BCVA in IogMAR units before ERM-ILM removal and the final postoperative BCVA in logMAR units (Additional Table S12).

In addition, the Spearman's rank correlation coefficient test showed a weak positive correlation $(r h o=0.32, p<0.05)$ between the preoperative period with the macula-off in weeks and the CSFT findings in microns; it also showed a weak negative correlation $($ rho $=-0.29, p<0.05)$ between the preoperative BCVA in logMAR units and ERM detection in weeks (Additional Figure S2).

The Mann-Whitney $U$ test comparing the peeling versus the non-peeling groups showed that the preoperative BCVA in logMAR units was statistically significantly different $(p<0.05)$ for the numeric variables of age, preoperative time period with the macula-off in weeks, BCVA in log MAR units before ERM-ILM removal, ERM detection in weeks, final postoperative BCVA in logMAR units, mean CSFT, and follow-up period in months (Additional Table S13).

The Mann-Whitney $U$ test showed that the postoperative BCVA in logMAR units was statistically significantly better $(p<0.05)$ for the numeric variables of age, preoperative period with the macula-off in weeks, BCVA in logMAR units before ERM-ILM removal, ERM detection in weeks, final postoperative BCVA in logMAR units, mean CSFT, and follow-up period in months.

The Mann-Whitney $U$ tests showed that the final BCVA in logMAR units after ERM proliferation removal was statistically significantly different $(p<0.05)$ for the numeric variables of age, preoperative time period with the macula-off in weeks, BCVA in log MAR units before ERM-ILM removal, postoperative ERM detection in weeks, final postoperative BCVA in logMAR units, CSFT alterations, and follow-up period in months.

The Kruskal-Wallis test showed that the preoperative BCVA in IogMAR units was statistically significantly different (Kruskal $\times 2=4.17, p<0.05$ ) with the ellipsoid band alterations when compared with the other variables (Additional Table S14 A). In addition, the postoperative BCVA in logMAR units was statistically significantly different $(p<0.05)$ among preoperative lens status, preoperative ERM, first surgery, recurrent RRD, additional surgery, postoperative ERM proliferation detection in weeks, foveal contour, presence of DONFL defects, mfERG, and microperimetry alterations (Additional Table S14 B). Furthermore, the final BCVA in logMAR units after ERM proliferation removal was statistically significantly different $(p<0.05)$ among the preoperative ERM proliferation, first surgery, recurrent $\mathrm{RRD}$, additional surgery, postoperative ERM proliferation detection, foveal contour abnormalities, DONFL defects, mfERG abnormalities, and microperimetry alterations (Additional Table S14 C).

The GLM for the preoperative BCVA in logMAR units showed that no variable was associated with the preoperative BCVA in log MAR units when adjusting for cofounders with multivariable analyses (Additional Table S15 A). It also showed (Additional Table S15 B) that the postoperative BCVA in logMAR units was significantly positively associated with the presence of significant ERM proliferation in the postoperative ERM proliferation analysis (coefficient $=0.45, \quad p<0.01$ ); significantly negatively associated when only vitrectomy (non-peeling 
group) was performed in the first surgery variable (coefficient $=-0.23, \quad \mathrm{p}<0.01$ ); and significantly negatively associated with the variable preoperative period of macula-off in weeks (coefficient $=-0.02$, $\mathrm{p}<0.05$; Additional Figure S3). In addition, the GLM for the final BCVA in log MAR units after ERM proliferation removal showed that it was significantly positively associated $(p>0.01)$ with the postoperative BCVA (Additional Figure S4), when only vitrectomy was the first surgery variable, and with the preoperative BCVA (Additional Figure S5) and male variable, when vitrectomy and ERM-ILM removal was the first surgery variable (Additional Table S15 C).

In the three groups in which a total of 230 eyes were analyzed, the general prevalence of preoperative primary ERM proliferation was 4.78\% (11 eyes), but only 10 eyes (4.34\%) underwent surgery; however, this prevalence should not be statistically considered due to the heterogeneity of criteria used to define a preoperative primary or postoperative secondary ERM proliferation and because the eyes without evidence of preoperative ERM proliferation were intentionally selected, and 10 out of 11 eyes detected with preoperative significant primary ERM proliferation were directly assigned to the peeling group.

The statistical program yielded the following SD-OCT abnormalities in the peeling group: ellipsoid band disruption was observed in 57.9\%, CSFT abnormalities in $94.7 \%$, ELM line alterations in $42.1 \%$, mfERG alterations in $89.5 \%$, and an abnormal microperimetry was detected in $78.9 \%$ of the eyes. In the non-peeling eye group, ellipsoid band disruption was observed in $21.3 \%$, CSFT abnormalities in 17\%, ELM line alterations in $31.9 \%$, abnormal mfERG in $8.5 \%$, and an abnormal microperimetry in $6.3 \%$ of the eyes (Table 2 ).

In the buckle group, the mean postoperative BCVA in logMAR units $(0.40 \pm 0.33 \mathrm{SD})$ was statistically significantly associated $(p<0.05)$ with the following variables: the presence of a significant postoperative ERM proliferation, the event of a retinal redetachment, ERM surgery, the presence of macular blood, and the event of ERM proliferation removal surgery. The GLM demonstrated that the final postoperative BCVA in logMAR units $(0.43 \pm 0.14 \mathrm{SD})$ after secondary ERM proliferation removal was statistically dependent on the following variables: postoperative BCVA after the first surgical procedure (buckle or primary vitrectomy), preoperative BCVA, and retinal perforation as a complication due to the buckling procedure and increased postoperative BCVA after ERM surgery by 0.15 logMAR units.

Analyzing the numeric variables mentioned with Wilcoxon rank-sum test and Fisher's exact test for the categorical variables (first surgery, BCVA before ERMILM complex removal, recurrence of RRD, additional surgery, ERM period detection, postoperative foveal contour appearance, DONFL defects, mfERG, and microperimetry alterations), we observed a statistically significant difference $(p<0.05)$ with better final BCVA in favor of non-peeling eye group (Additional tables S2 and S11). We used one-factor ANOVA test to compare the postoperative BCVA with the buckle group, the nonpeeling group, and the peeling group, and the resultant $p$-value was $0.001(p<0.05)$.

In the non-peeling group, we compared postoperative BCVA and abnormal findings on OCT (ellipsoid band, CSFT, ELM line). When comparing the ellipsoid band as a biomarker with the postoperative BCVA in logMAR units, student's t-test was performed, resulting in a $p=0.001$, with a Pearson correlation coefficient of 0.314; hence, a larger value of logMAR was associated with more ellipsoid band disruptions. Further, we compared CSFT with postoperative BCVA in logMAR units, and performed Student's t-test, we obtained the $p$-value as $0.001(p<0.05)$, with a Pearson correlation coefficient of 0.403 ; hence, a higher BCVA in log MAR units was associated with more CSFT abnormalities. Similarly, on comparing ELM with postoperative BCVA in logMAR units and performing Student's t-test, we obtained the $p$-value as $0.001(p<$ 0.05), with a Pearson correlation coefficient of 0.192 , showing that a higher logMAR was associated with a greater presence of ELM line abnormalities.

The above analyses also applied to those eyes in the peeling group after ERM proliferation removal complemented with ILM removal. On comparing ellipsoid band disruptions with postoperative BCVA in logMAR units, and subsequently performing the Student's t-test, we obtained the p-value as 0.001 $(p<0.05)$ and a Pearson correlation coefficient of 0.061. We observed that a higher value of BCVA in logMAR units was associated with more ellipsoid band disruptions.

On comparing CSFT alterations with postoperative BCVA in logMAR units, the Student's t-test showed $p$-value of $0.001 \quad(p<0.05)$ and a Pearson correlation coefficient of 0.13 . Thus, we observed that a higher value of logMAR was associated with more CSFT alterations.

The relationship of ELM line alterations and postoperative BCVA in logMAR units showed a Student's t-test result of $p=0.001 \quad(p<0.05)$ and a Pearson correlation coefficient of -0.102 . In this case, we observed that a higher BCVA in logMAR units was associated with a lower incidence of ELM line alterations in the SD-OCT.

A correlation was sought between the presence of DONFL defects (dimples) in the peeling group according to the postoperative BCVA in logMAR units. In the non-peeling group, no eyes developed dimples regardless of their BCVA. In the peeling group, the mean postoperative BCVA in logMAR units of eyes that did not have dimples was $0.52 \pm 0.14 \mathrm{SD}$, and the mean postoperative BCVA in logMAR units of eyes that 
developed dimples was $0.59 \pm 0.16 \mathrm{SD}$. A necessary comparison of these values was performed to check if the data came from a normal distribution. Hence, the Shapiro-Wilk test was performed, which resulted in 0.89; therefore, coming from a normal distribution, Student's ttest was performed for independent samples, which resulted in $p=0.32 \quad(p>0.05)$, thereby indicating the absence of a statistical significance.

The postoperative BCVA in logMAR units in the peeling group that did not have ERM proliferation according to the SD-OCT was analyzed and correlated; in this way, no statistical significance was detected in the vision between the eyes with and without SD-OCT abnormalities such as ellipsoid band disruptions $(p=0.848, \quad p>0.05$, respectively), CSFT alterations $(p=0.05)$, ELM line abnormalities $(p=0.653, p>0.05)$, mfERG abnormal findings $(p=0.74, p>0.05)$, and microperimetry alterations $(p=0.20, p>0.05)$.

The same comparisons were made in the nonpeeling eye group who developed ERM proliferations. The BCVA in logMAR units correlated with the presence of ellipsoid band abnormalities, ELM line abnormalities, and mfERG alterations, and microperimetry abnormalities was compared with those of eyes without such defects; we did not find any significant differences $(p>0.05)$.

Further, the same groups were compared but without consideration to the presence of an ERM proliferation, a positive statistical significance $(p<0.05)$, and BCVA correlation, when CSFT, mfERG abnormalities, and microperimetry alterations were comparatively analyzed between eyes with and without these abnormalities.

Moreover, microperimetry and mfERG revealed abnormal retinal responses with a stable but extrafoveal (eccentric) fixation pattern, a profound reduction in N1and $\mathrm{P} 1$-wave $\mathrm{nV}$ amplitudes, and a prolonged $\mathrm{P} 1$ implicit time predominantly in the ILM peeling group. The functional responses were predominantly normal in the buckle and non-peeling groups without postoperative ERM proliferation.

Finally, in the peeling group, there was neither statistical significance $(p=0.819, p>0.05)$ nor visual correlation when the postoperative BCVA in logMAR units was compared between eyes with the presence of DONFL defects and those without it.

In the buckle eye group, more additional surgeries were needed for complications such as recurrent RRD (11 eyes) with an additional surgery rate (ASR) of $8.8 \%$, ERM-ILM complex removal (27 eyes; ASR of $21.6 \%$ ), buckled revision (4 eyes; ASR of 3.2\%), phaco-vitrectomy (3 eyes; ASR of 2.4\%), vitrectomy (2 eyes; ASR of $1.6 \%)$, phaco-vitrectomy ERM-ILM complex removal (1 eye; ASR of $0.8 \%$ ), vitrectomy ERMILM complex removal (1 eye; ASR of $0.8 \%$ ), and other serious surgical complications that were treated conservatively and without surgery such as through and through complication drainage phenomenon (8 eyes; $6.4 \%$ ), retinal perforation (7 eyes; $5.6 \%$ ), transoperative presence of submacular blood as a complication of SRF drainage or full-thickness scleral perforations (5 eyes; 4.0\%) handled with pneumatic displacement, and noncomplex vitreoretinal entrapment released with surgical maneuvers in the first surgery (3 eyes; $2.4 \%$ ), with a general ASR of $37.6 \%$ in the buckle group (Additional Table S3). The ASR seen in the vitrectomy group was $9.6 \%$ (12 eyes), with vitrectomy revision in 9 eyes (8.6\%), only vitrectomy 2 eyes (1.9\%), and phako-vitrectomy ERM-ILM peeling 1 eye (1.0\%). The comparative incidence of early or short-term postoperative complications between the buckle group and the vitrectomy groups that required additional surgical procedures was statistically significant $(p<0.05$ Student's t-test).

\section{Discussion}

Skill and practice are needed to place a scleral buckle in the correct location with the desired indentation to support the vitreous base and retinal tears and to drain transscleral SRF without complications. The use of vitrectomy techniques has expanded greatly nowadays owing to unprecedented advances in vitrectomy platforms, development of more rigid smallgauge cutters with improved fluidics and better instrumentation, and the widespread availability of wideangle viewing systems with superior endoilluminators. Some studies suggested that vitrectomy techniques alone should be employed in the management of a simple, primary, non-complicated macula-off RRD. While some cases can be managed successfully with vitrectomy, an important subset of non-complicated, macula-off RRD will benefit from buckling techniques. All surgical approaches in this retrospective report were performed to achieve the patient's best interest and to determine the best technique for particular circumstances of RRD. To achieve these, we retrospectively analyzed the charts of scleral buckling techniques in 125 consecutive selected eyes which fulfilled the inclusion criteria and primary vitrectomy or vitrectomy without and with ILM removal in 105 selected eyes which also fulfilled the inclusion criteria that were treated for non-complicated macula-off RRD; we conducted a retrospective, long-term, multicenter, onesurgeon, comparative structural and functional macular evaluation (Figure2 control normal eye; images 1A-1A$6)$; further, we reported our experience of the real-life postoperative incidence of ERM proliferation over the macula and statistically intercorrelated those findings across the groups. The study aimed to evaluate the main complications of buckling surgery (Figure3 C-P images) and vitrectomy (Figure4 A-H-2 images; Figure5 $\mathrm{I}-\mathrm{T}$ images) among the groups. 


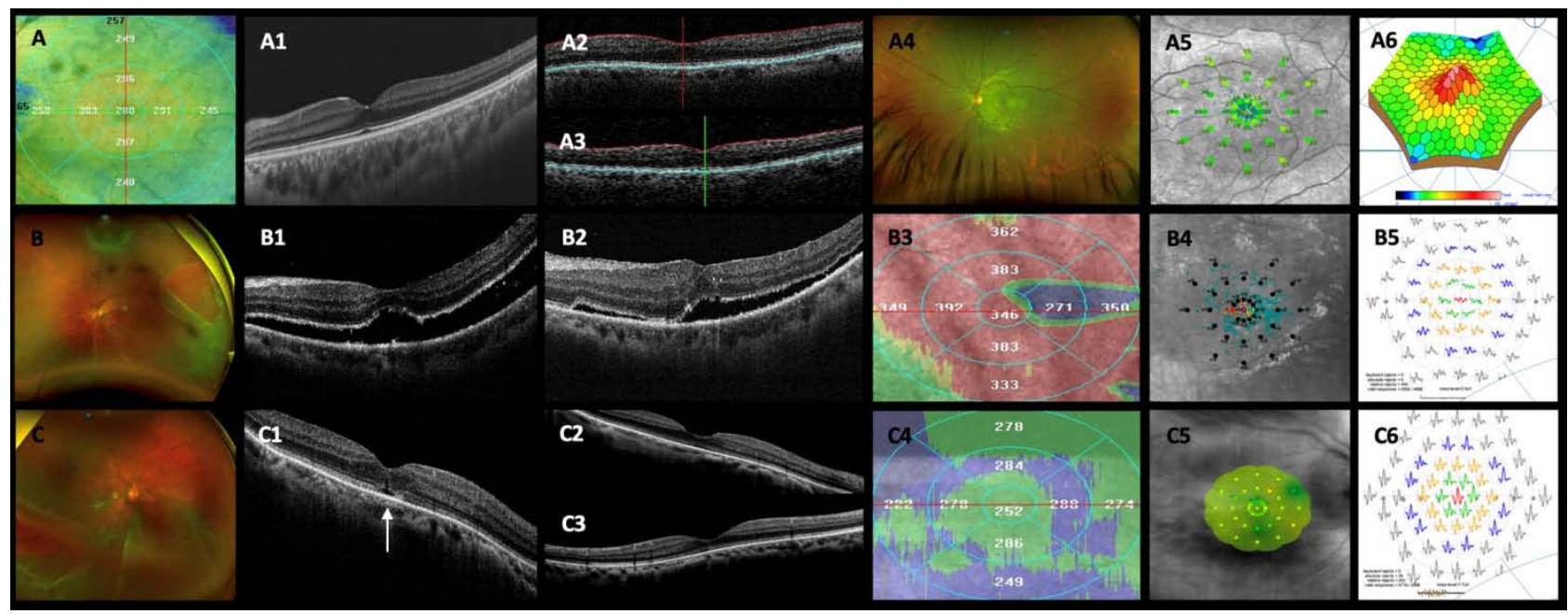

Figure 2: Postoperative structural and functional findings (part 1).

(A)-(A-5) Normal control eye. (B) Primary rhegmatogenous retinal detachment (RRD); large posterior rolled edge retinal tear at 2 o'clock meridian managed with primary vitrectomy (case 2; non-peeling group). (B-1) Spectral-domain optical coherence tomography (SD-OCT) horizontal scan with postoperative subretinal fluid (SRF) 3 weeks after vitrectomy. (B-2) Shallow amount of SRF 8 weeks later. (B-3) Abnormal topographic thickness retinal map on Ret-vue SD-OCT with diffuse retinal thickening. (B-4) Macular microperimetry showing eccentric foveal fixation. (B-5) Corresponding multifocal electroretinogram (mfERG) depicting abnormal electrical response in three central rings with the $\mathrm{nV}$ decreased; final best-corrected visual acuity (BCVA) is 0.18 logarithm of the minimum angle of resolution (logMAR) units. (C) An Optos photo showing primary RRD involving the macula; arrow-shaped retinal tears are seen at 7 o'clock, and there is preoperative epiretinal membrane (ERM) proliferation (case 87 ; peeling group). (C1) SD-OCT image 8 weeks postoperatively depicting defects in the ellipsoid and external limiting membrane (ELM) disruption (white arrow). (C-2) and (C-3) Macula crossline scans, with an ellipsoid and ELM biomarkers recovered. (C-4) Postoperative normal topographic thickness macula map after undergoing a successful, two-step ERM- internal limiting membrane (ILM) removal technique. (C-5) Macular microperimetry with macula retinal sensitivity, foveal retinal sensitivity, and a stable foveocentral fixation pattern; the retinal sensitivity analysis map shows normal macular integrity at the end of the follow-up. (C-6) mfERG of the corresponding macular area. The P1 implicit time is normal in the <2-degree central ring and slightly longer in the remaining central rings. The $\mathrm{nV}$ amplitude in the normal range is comparable to the normal age-matched control eye, and the BCVA is 20/40 (0.30 logMAR units). (D)-(D-5) Sequence of macular ERM-ILM two-step removal technique events. 


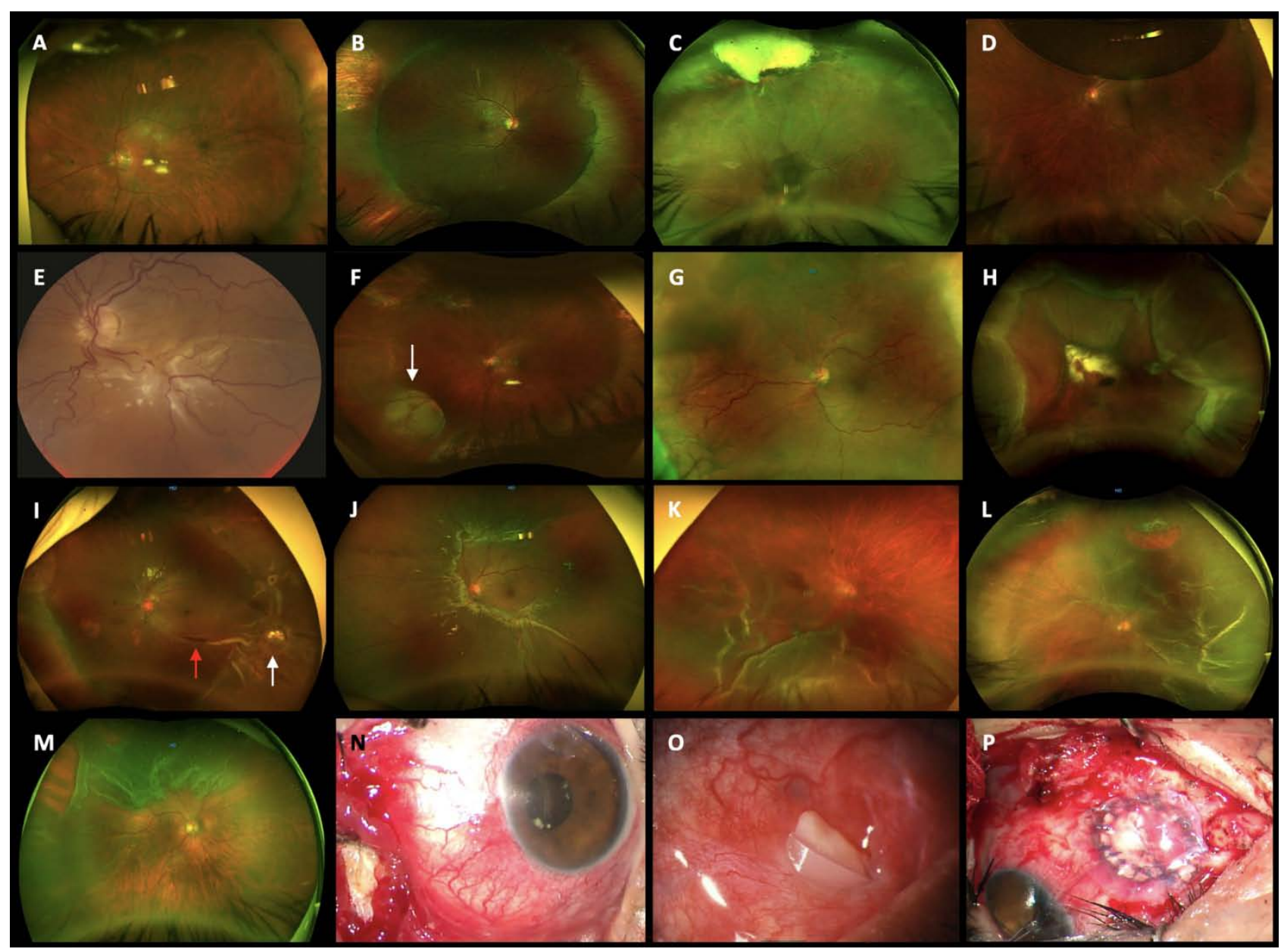

Figure 3: Transoperative and postoperative buckle complications.

(A) and (B) Buckled rhegmatogenous retinal detachment (RRD) (cases 4 and 58); final best-corrected visual acuity (BCVA) is 0.18 logarithm of the minimum angle of resolution (logMAR). (C) Whitish condensed vitreous hemorrhage; a recurrent RRD is detected; the eye underwent vitrectomy. Six weeks after, retina remains attached with epiretinal membrane (ERM); logMAR is 0.60 (D) Buckled eye with 15\% sulfur hexafluoridegas; there is a tear at the 5 o'clock with RRD (case 88); after positioning and laser, the vision is 0.30 logMAR. (E) Buckled eye with recurrent RRD (case 15); a retinal fold resolved with ERM-internal limiting membrane (ILM) removal; final BCVA logMAR is 0.40 (F) Case 19. A postoperative granuloma (white arrows) 6 weeks after surgery; a low-grade inflamed course persists; presence of a dome-shaped granuloma (white arrow) at the 7 o'clock meridian, which resolves periocular antibiotics; final logMAR was 0.18is 20/30. (G) Choroidal hemorrhagic detachment after scleral perforation. (H) A 360-degree, non-kissing, hemorrhagic choroidal detachment after a complicated scleral buckle procedure. (I) Vitreoretinal entrapment (white arrow) with retinal fold and preretinal blood (red arrow); BCVA is 20/100 (0.70 logMAR). (J) An Optos photo depicts a recurrent RRD 6 weeks after a buckling procedure (case 112); there is ERM proliferation and PVR over the posterior pole; ERM-ILM removal was performed; final logMAR was 0.60. (K) Scleral perforation; submacular blood displacement is required, and the eye has undergone ERM-ILM removal; BCVA is 0.60 logMAR (case 93). (L) leaking retinal tear; the eye undergoing phaco-vitrectomy; final BCVA is 0.30 logMAR (case 54). (M) A failed buckling (case 43) with a rolled posterior edges retinal tear; ERM proliferation removal; BCVA is 0.40 logMAR. (N) Sponge exposition 32 months after surgery (case 40). (O) Hard silicone extrusion. $(P)$ Scleral patch and amnios graft for buckle-related scleral thinning. 

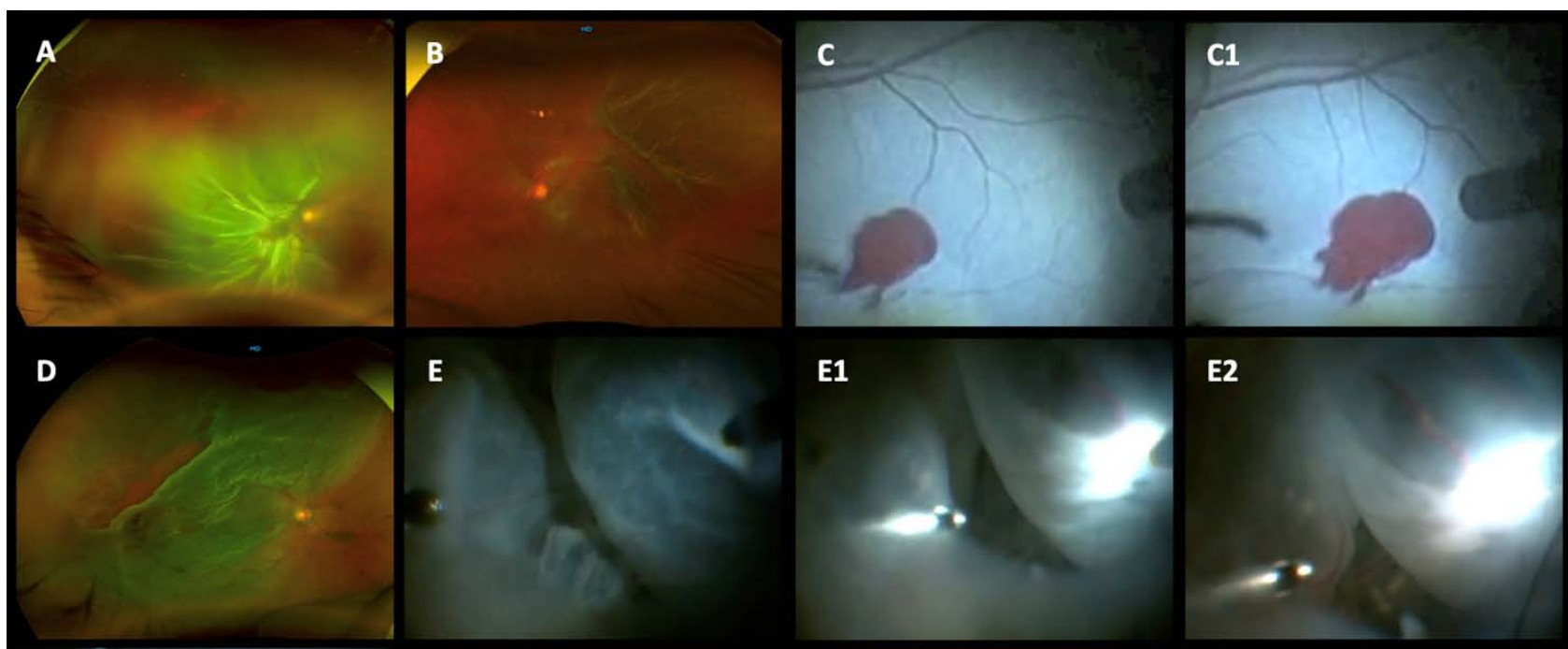

E

E1

E2
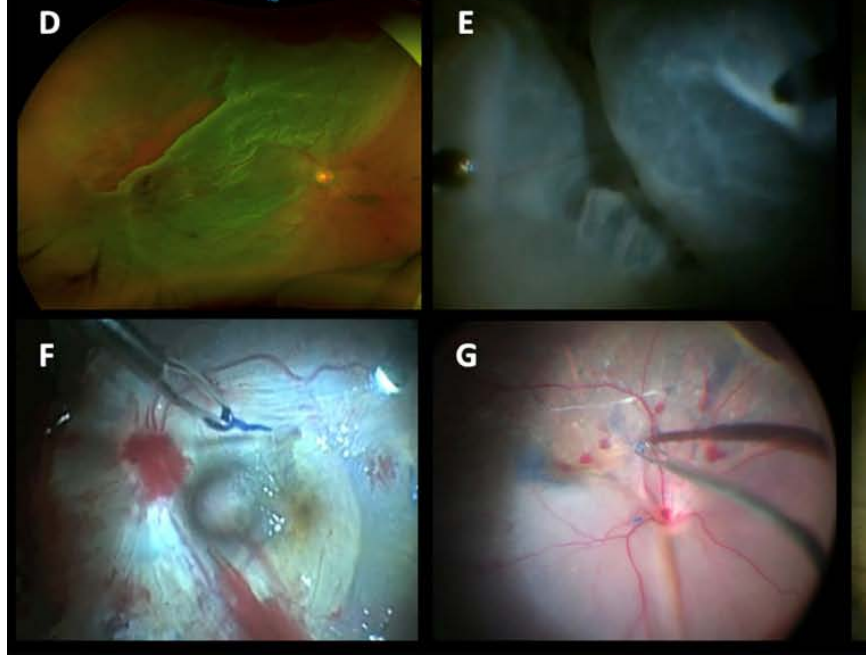

G

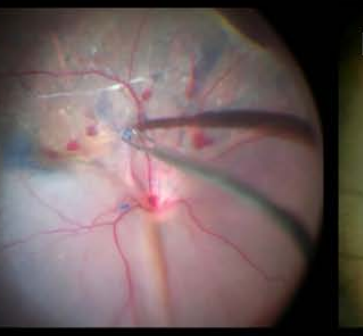

H

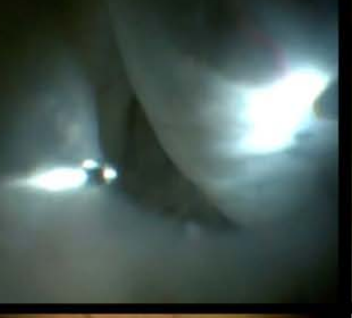

4
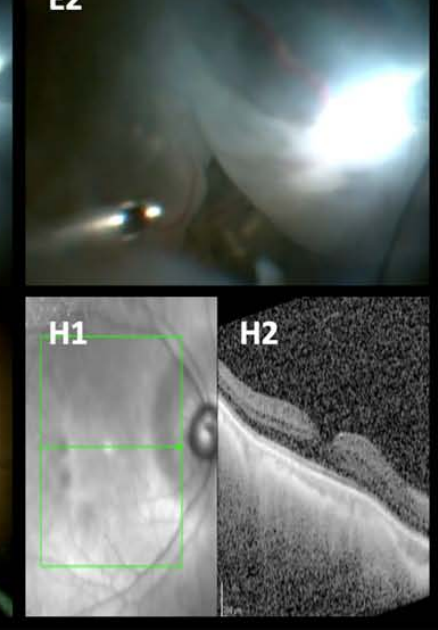

Figure 4: Transoperative and postoperative vitrectomy complications (part 1).

(A) Retinal detachment complicated by posterior proliferative vitreoretinopathy; the retina is totally detached, and the macula appears contracted due to the presence of diffuse epiretinal proliferation. (B) An Optos, color photo of are current rhegmatogenous retinal detachment (RRD) in a failed primary vitrectomy; there is no gas tamponade inside the eye, and the retina is detached mainly over the posterior pole with the macula off; the patient undergoing vitrectomy revision with laser endophotocoagulation. $(\mathrm{C})$ and $(\mathrm{C}-1)$ A rather dim brilliant Blue $\mathrm{G}(\mathrm{BBG})$ internal limiting membrane (ILM) staining with arterial bleeding at the time of pulling the ILM in a case of a shallow macula-off retinal detachment; this complication is resolved by raising the transoperative intraocular pressure for a few minutes. (D) Complicated RRD 3 weeks after a failed gas-vitrectomy and epiretinal membrane-ILM removal procedure; the retina looks rigid, and there is a large tear with a posterior rolled edge. (E)-(E-2) Sequential hydraulic choroidal and retinal detachment as a transoperative complication due to erroneous positioning of the infusion cannula; the hydraulic complication grows progressively as the cut and suction instrument is working, and by changing the entrance of the infusion cannula, the complication is resolved. (F) Bleeding from the papilla as we peel off the ILM in this macula-off RRD case; in this case, BBG ILM staining-perfluorocarbon heavy liquids are used to reapply the retina-ILM peeling; (G) Tractional bleeding at the moment of the ILM being pulled to release the macula. $(\mathrm{H})$ Multiple spots bleeding due to inner punctate hemorrhagic retinopathy related to ILM peeling. $(\mathrm{H}-1)$ and $(\mathrm{H}-2)$ En-face superficial imaging of the presence of dark, well-delineated, superficial retinal spots compatible with dissociated optic nerve fiber layer defects; in this case, there is no evidence of superficial dimples on the corresponding Spectralis horizontal spectral-domain optical coherence tomography. 


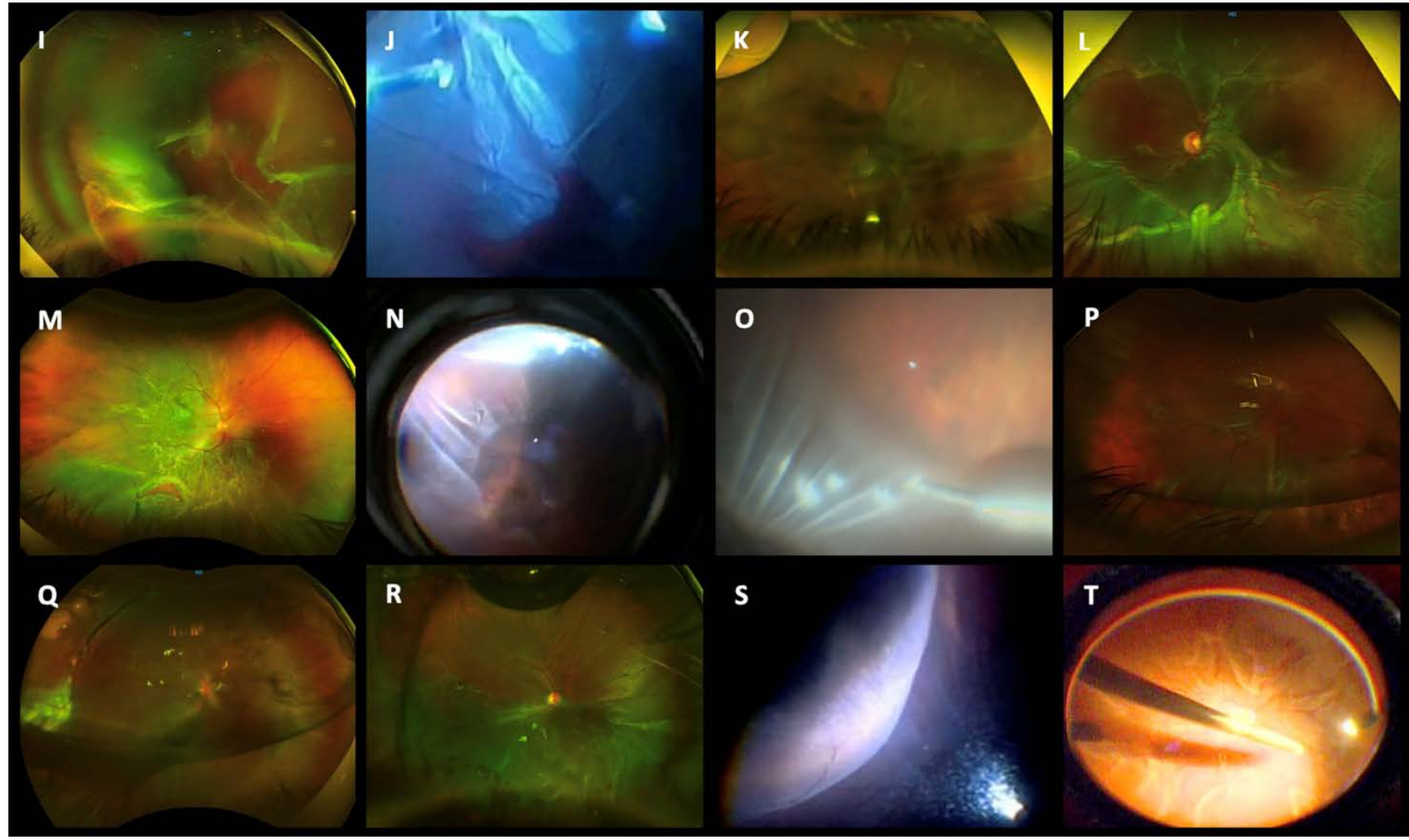

Figure 5: Transoperative and postoperative vitrectomy complications (part 2).

(I) An Optos, wide-angle, color fundus depicts a hemorrhagic choroidal rhegmatogenous retinal detachment (RRD) detected 3 days after primary vitrectomy. (J) Highly complex vitreoretinal entrapment at the level of superior trocar sclerotomy due to undetected transient eye hypotony secondary to transoperative surgical manipulation of the retina. (K) Evidence of vitreous, choroidal, and subretinal bleeding with the persistence of RRD. (L) An Optos, wide-angle, color photo 6 weeks after primary vitrectomy with proliferative vitreoretinopathy (PVR) complicated by RRD as a late vitrectomy complication in the management of primary, non-complicated RRD. (M) Subtotal RRD after primary vitrectomy; an active, leaking retinal tear with rolled-back borders can be seen between the 6 and 7 o'clock meridians; there is evidence of macular rigidity and contraction due to the presence of diffuse epiretinal membrane (ERM) retina proliferation. (N) Transoperative vitreoretinal entrapment at the level of the entry vitrectomy site; an active leaking arrowed-shaped retinal tear is observed at 11 o'clock meridian at the equator zone. (O) shows another transoperative image with a vitreoretinal entrapment at the entry vitrectomy infusion site. $(P)$ shows a low-grade illumination transoperative step of a recurrent complicated PVR case after primary vitrectomy. (Q) A failed buckling of recurrent RRD that has undergone ERM-internal limiting membrane (ILM) complex removal due to significant ERM macular proliferation; there are some recent argon laser spots and a $70 \%$ residual sulfur hexafluoride $\left(\mathrm{SF}_{6}\right)$ gas bubble with a shallow recurrent retinal detachment. (R) Recurrent inferior RRD after primary vitrectomy with residual $\mathrm{SF}_{6}$ gas bubble. (S) Hydraulic choroidal and pars plana detachment is caused by mispositioning of the infusion line of the trocar entry sclerotomy site. (T) shows a total recurrent RRD in a pseudophakic eye 30 days after primary vitrectomy with ERM-ILM complex removal due to significant macular ERM proliferation managed with the in-block ERM-ILM technique.

In cases such as those described in the vitrectomy groups in this study, we believe that adding a buckle is unnecessary and adds additional risk and possible undesirable postoperative complications and cost to an already sophisticated procedure; hence, to analyze the complications of scleral buckling (Figure3 $\mathrm{D}-\mathrm{P}$ ) and vitrectomy techniques (Figures4 A-H-2 images; Figure5 I-T images), only eyes without a supplemental scleral buckle were included in the final statistical analyses. The management of noncomplicated RRD with scleral buckling was compatible with good anatomic outcomes (Figures3 A and B images); however, this procedure can be associated with transoperative and postoperative complications (Figure3 C-P images), leading to performing additional surgery. Hence, we included only eyes with noncomplicated macula-off RRDs, analyzed their management and incidence of complications retrospectively, and compared the incidence of postoperative ERM proliferation and surgical complications as well as structural and functional findings in all three groups (Figure2 B-B5, C-C6; Figure1 E-E6, F-F3; Figure6 A-A5, B-B6). Currently, in the management of macula-off RRD with vitrectomy, we placed a supplemental $360^{\circ}$ scleral buckle only in complex or complicated cases involving diffuse tractional membranes such as RDs complicated with significant PVR, failed prior RRD surgery, extensive peripheral vitreoretinal adhesions with multiple retinal tears, RRD associated with penetrating globe-injury 
and/or retain intraocular foreign body, and selected RRD associated with giant retinal tears.

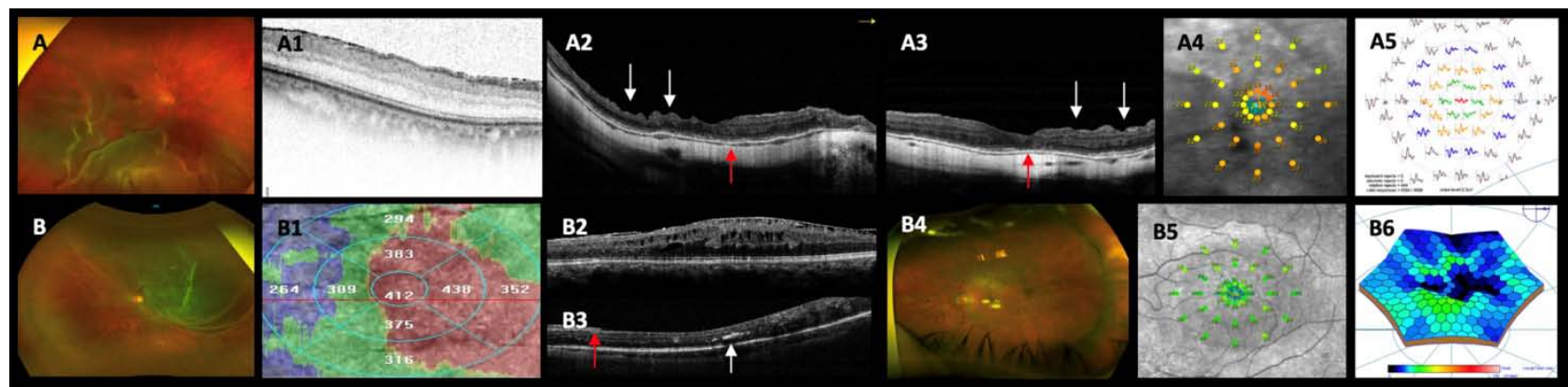

Figure 6: Postoperative structural and functional findings (part 3).

(A) Optos photo of a failed gas-vitrectomy in rhegmatogenous retinal detachment (RRD)(case 78). (A-1) Corresponding macula scan after vitrectomy revision with postoperative epiretinal membrane proliferation with logarithm of the minimum angle of resolution of 1.0 units; the eye underwent a third procedure for ERM-internal limiting membrane removal; final BCVA is 0.70 logMAR. (A-2) and (A3) Foveal crossline depict retinal thinning and dimpling of the superficial nerve fiber layer (white arrows) due to dissociated optic nerve fiber layers defects and loss of foveal contour; the ELM line and ellipsoid show irregular reflectivity (red arrows). (A-4) Macular microperimetry with a reduction in macular retinal sensitivity (MRS); the retinal sensitivity analysis shows abnormal integrity. (A-5) Abnormal multifocal electroretinography (mfERG) response with $\mathrm{N} 1$ wave amplitude reductions. (B) An Optos photo of a primary RRD undergoing an uneventful 360-degree 503 round sponge buckling-cryotherapy with subretinal fluid drainage; After 6 weeks, macular thickening associated with epiretinal membrane (ERM) proliferation is depicted in (B-1), with diffuse retinal thickening in the abnormal topographic retinal map.(B-2) Corresponding horizontal scan depicting diffuse macular thickening associated with ERM proliferation and wrinkling of the inner superficial retina layers; multiple deep cystic spaces (sponge-type) and submacular fluid are seen; although the ellipsoid band appears preserved, the ELM line is not; final BCVA is 0.90 logMAR. (B-3) After ERM-ILM removal, the BCVA is 0.70 logMAR. The macula looks atrophic; there are hyper reflective deep lines (white arrow) and ILM remnants (red arrow); no evidence of the ELM and ellipsoid are found. (B-4) An Optos, wide-angle photo of the corresponding cases shows substantial, but non-significant, visual improvement (case 43; peeling dataset). (B-5) The corresponding microperimetry shows subnormal macular integrity with subnormal MRS; stable foveocentral fixation is seen.(B-6) Abnormal three-dimension topographic map of the corresponding abnormal mfERG.

Although the use of a supplemental scleral buckle has evolved throughout the years, its selective use seems to be compatible with good outcomes in non-complicated cases[14]. However, in complex cases with total RD, significant PVR, and posterior insertion of the vitreous base, additional scleral depression to reach the pathological vitreous base to facilitate its dissection must be performed to facilitate vitreous base shaving and release vitreous traction at this level, in addition to the proper placement of a $360^{\circ}$ scleral cerclage. Other surgical maneuvers that are considered extreme, such as circumferential retinotomy and retinectomy, are rarely performed[15]; this is because vitrectomy has a better anatomical outcome in such complicated cases when complemented with scleral buckling.

Some surgeons peel the ILM off only if there are pre-existing ERM proliferations in the macula[16], as we reported in the peeling vitrectomy group, while others never perform routine ILM peeling to prevent postoperative ERM proliferation and prefer its removal during a second surgery if there is ERM proliferation occurrence and according to the sight evolution[15-17], this means that they will need an additional vitrectomy procedure only if they are highly symptomatic or show significant structural and functional alterations in the macula due to the secondary postoperative presence of ERM proliferation. The incidence of postoperative ERM proliferation has been reported to range from $27.6 \%$ to $38.4 \%$ after cryoretinopexy and from $21.5 \%$ to $58 \%$ after vitrectomy without ILM removal.[12,17,18] Herein, we reported a postoperative ERM proliferation incidence of $23.2 \%$ (29 eyes) in the buckle group, 23.63\% (13 eyes) in the non-peeling group, and $2.0 \%$ (one eye) in the peeling group (Figure1 E1-E2 and F-F3 images).

A previous prospective and comparative study[19] did not identify any functional or structural benefits of ILM peeling during primary vitrectomy for non-complicated RRD; the authors showed a very low incidence $(0.003 \%)$ of significant ERM in eyes where ILM peeling had been performed and found that these patients had a lower final BCVA than those whose ILM had not been removed (mean logMAR units BCVA $1.0 \pm 0.4$ vs. $0.4 \pm 0.2, \quad p<0.001)$; these functional findings were also found in our study. However, in a retrospective report, Garweg et al.[20] described an unprecedented visual gain over 6 months after successful primary reattachment surgery with peeling of the ILM and sulfur hexafluoride gas tamponade, which did not show the same results as the peeling group in our study. Some authors [19] and the authors of the present study agree that although ILM peeling prevents ERM, it results in a poorer visual outcome in such noncomplicated macula-off RRD cases and may therefore be better reserved only for selected complicated cases. 
In this study, in the vitrectomy groups, we found that some variables, such as first surgery, BCVA before ERM-ILM removal, recurrence of ERM, additional surgery (Figure1 F-4, F-5), ERM proliferation detection in weeks, final postoperative BCVA, foveal contour abnormalities (Figure 4 D-3 image), DONFL defects (Figure $4 \quad \mathrm{C}-2$ and $\mathrm{C}-3$ images), mfERG and microperimetry findings alterations (Figure 7 C-4 and C-5 images) were more common in the peeling group than in the non-peeling group with significant statistical differences $(p<0.05)$ between the peeling and nonpeeling groups analysis (Additional Table S5). However, the functional analysis of these structural abnormalities in SD-OCT considered as categorical variables such as ellipsoid band disruptions, CSFT abnormalities, and ELM line discontinuities could not be found a direct correlation with the final BCVA due to a lack of statistical significance.
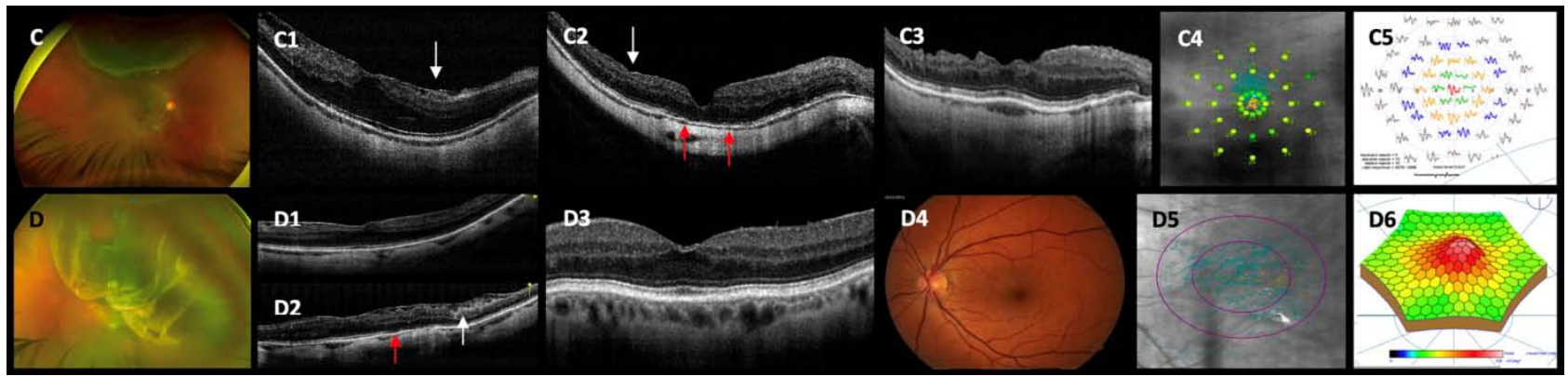

Figure 7: Postoperative structural and functional findings (part 4).

(C) An Optos photo showing rhegmatogenous retinal detachment with epiretinal membrane proliferation (case 23). (C-1) Horizontal foveal scan with macular thickening and subretinal fluid; ERM proliferation is shown (white arrow); foveal thinning and loss of the external limiting membrane (ELM) line with ellipsoid band reflectivity attenuation. (C-2) Horizontal foveal scan of the foveal contour with a thin fovea; dimples are seen (white arrow); ELM looks discontinuous, and the ellipsoid band shows hyporeflectivity (red arrows). (C-3) After 4 months,the vertical foveal scan depicts normal central subfoveal thickness (CSFT); there is loss of the foveal contour and dimpling of the superficial retinal layers. The ELM line and ellipsoid band look normal. (C4) Abnormal macular microperimetry. (C5) Multifocal electroretinography abnormal electrical response. (D) An Optos photo with a bullous pseudophakic RRD (case 6; peeling group) undergoing primary vitrectomy; After 8 weeks, ERM proliferation is depicted on the foveal crossline scan. (D-1) and (D-2) ERM proliferation is clearly seen (white arrows) along with wrinkling of the superficial retina. At this stage, the ELM line looks discontinuous, and the ellipsoid shows disruption (red arrow); the best-corrected visual acuity (BCVA) at this point is 0.48 logarithm of the minimum angle of resolution (logMAR). (D-3) After 12 weeks after, a normal foveal contour is depicted within a normal CSFT; there is a hyper reflective horizontal line below the fovea and a non-cyst hypo reflective space. The reflectivity of the ELM line appears attenuated with discontinuation and a recovered ellipsoid. (D-4) Corresponding color fundus without relevant clinical macular details. (D-5) Abnormal microperimetry, with a central fixation pattern; the retinal sensitivity analysis map shows normal integrity. (D-6) Normal three-dimension topographic macular map compared with the normal matched-age normal control; the final BCVA after ERM-internal limiting membrane complex removal is $0.30 \log M A R$ at the end of follow-up.

Herein, we studied 230 consecutive selected eyes and retrospectively analyzed the cases, and we found functionally unsatisfactory results in the ILM peeling group compared with those in the buckle and non-peeling eye groups. The postoperative BCVA in $\log M A R$ units was significantly associated $(P<0.05)$ with the following variables: the presence of significant postoperative ERM proliferation, retinal redetachment, the presence of submacular blood, and the event of ERM proliferation surgery, which means that the presence of any of these variables significantly influences the final visual result. The GLM showed that postoperative BCVA was statistically significantly correlated with the following variables: postoperative ERM proliferation, which increased the postoperative BCVA by 0.68 in logMAR units, and retinal entrapment, which decreased the postoperative BCVA by 0.21 in logMAR units. The GLM also showed that the final postoperative BCVA after ERM surgery was statistically significantly dependent on the following variables: postoperative BCVA in logMAR units, preoperative BCVA in logMAR units, and retinal perforation event, which increased the postoperative BCVA after ERM surgery by 0.15 in logMAR units.

The anatomical results regarding successful reattachment of the retina were satisfactory in the three groups; however, we found a significant percentage of postoperative ERM proliferation in the buckle and in the non-peeling groups. Although we found only one eye with postoperative ERM proliferation in the peeling group, we observed multiple structural alterations in the SD-OCT biomarkers, as mentioned earlier, along with multiple functional alterations with a significant reduction in retinal sensitivity. The macular mapping using microperimetry showed excentric with stable fixation patterns in most of the eyes studied; we also observed a significant reduction in the mean MRS and mean FRS at the four central points, starting from the central $2^{\circ}$, as well as an abnormal mean retinal sensitivity analysis map in all the peeling cases studied compared with the 
buckle, non-peeling, and normal control eye (Figure2 A5, B-4, and C-5 images; Figure 6 A-4 and B-5 images; Figure7 C-4 and D-5 images). We found that the threedimensional mfERG map was abnormal in most of the peeling cases studied, and the electric tracing showed a significant mean reduction in the N1-wave amplitude and prolonged implicit times in P1 waves, indicating low activity of bipolar cells and photoreceptor and inner retinal ganglion cells dysfunction (Figure2 B-5 and C 6 images; Figure1 E-6 image; Figure6 $\mathrm{A}-5$ and B-6 images, Figure7 C-5 image). Notably, the eyes that developed secondary postoperative ERM proliferations in the buckle group and in the non-peeling group showed statistically significant upgrading in BCVA once the macular ERM proliferation was removed, but the abnormalities in the status of the SD-OCT biomarkers, mfERG, and microperimetry did not disappear, as shown in the serial analyses of some of our clinical cases.

Only one study [17] has reported the role of prophylactic ILM removal in reducing the incidence of postoperative ERM proliferations, and few studies have correlated ILM removal with serial or Iongitudinal findings such as the status of biomarkers from SD-OCT and serial functional results obtained using computerized mfERG and microperimetry [21,22]. Similar to previous studies, we found limited benefits of ILM removal; although there was a significant postoperative reduction in ERM proliferations, this did not justify implementing this technique on a regular basis.

Although this approach avoids new surgical procedures and the patient can be kept free of macular symptomatology, ILM removal is not without potential transoperative complications, such as those related to mechanical trauma, including retinal tears, retinal edema, papilar hemorrhage (Figure 4 F), retinal hemorrhage (Figure $4 \mathrm{~F}, \mathrm{G}$ ), iatrogenic punctuate hemorrhagic retinopathy (Figure4 $\mathrm{G}$ and $\mathrm{H}$ image), vitreous hemorrhage (Figure $4 \mathrm{C}-1$ and $\mathrm{C}-2$ images), subretinal hemorrhage; and postoperative late functional findings such as excentric fixation patterns (Figure2 B-4; Figure1 E-5), microperimetric abnormal macular integrity with subnormal macular retinal sensitivity (Figure6 B-5), or central scotomas of different densities described by other[23]; most of them are at the subclinical level but favoring poor quality vision and poor final BCVA recovery. Moreover, possible structural sequelae such as DONFL defects may occur because of a diffuse loss of Muller cell end-feet[22,24,25]. In this study, a DONFL defect appearance in the form of concentric macular dark spots (Figure $4 \mathrm{H} 1$ and $\mathrm{H}-2$ images), known as retinal dimples, was detected in our clinical cases only in the postoperative, long-term SD-OCT evaluations of the peeling eye group (Figure6 $\mathrm{A}-2$ and $\mathrm{A}-3$ images; Figure7 C-1, C-2, and C-3 images), and in some eyes, modified and improved appearance of the external layer of the retina was observed without a total recovery of the normal tomographic pattern (Figure2 C-1-C-3 images). This superficial dimpling finding had been reported as a consequence of ILM removal and was first described by Alkabes et al.[25]as a subclinical finding; its effect on macular function as measured by microperimetry and mfERG [22] is still controversial. Our results revealed that the presence of alterations in the microperimetry and mfERG had no statistically significant correlation with the final BCVA when comparing the eyes with the presence or absence of these DONFL defects (dimples) findings (Figure6 A-2 and A-3 images; Figure7 C-1-C-3 images; Figure $4 \mathrm{H}-1$ and $\mathrm{H}-2$ images) nor was statistically significant or statistical correlation was found between the number of dimples and the final visual acuity. We do not know yet how these changes in the retinal nerve fiber layer affect the macular function or how they can impact and correlate with postoperative visual recovery.

The functional analysis correlated with the presence of DONFL defects indicated that in the peeling group, the mean postoperative logMAR units BCVA in the patients who did not have dimples was $0.52 \pm 0.14$ $\mathrm{SD}$, the mean postoperative logMAR units BCVA in those who developed dimples was $0.59 \pm 0.16 \mathrm{SD}$, and the $p$-value was 0.89 ; Student's t-test was $p=0.32$ ( $p$ $>0.05$ ), indicating no statistical significance, meaning that, clinically, the presence of DONFL defects due to the removal of MLI does not appear to have functional repercussions on the final BCVA as previously described by other authors[21]. These defects were not evident when MLI was not removed as we were able to verify this fact in the buckling and in the non-peeling groups; when the ILM is removed, the final BCVA is practically the same in the eyes that develop defects and in the eyes that do not develop them.

In contrast, other studies [26] have shown that the final BCVA correlates better with the time period the photoreceptors remain detached from the RPE. This possible deleterious complication might be correlated with the appearance of the ellipsoid band zone, and this strong SD-OCT biomarker was found to be serially abnormal and disrupted in our study. Schuman et al (27) tried to correlate histopathologically the retinal cleavage plane of the ILM using transmission electron microscopy with the functional results, there was no conclusive remarks if the presence and amount of retinal cell fragments at ILM specimens correlate with functional deficits.

Furthermore, only one (2.0\%) out of 50 eyes in the peeling group in this study was found to harvest long-term residual SRF; however, advanced age is considered a significant risk factor for the development of postoperative SRF, especially in patients where the ILM is removed. A gradual decrease in RPE pumping due to aging after reattachment to the neurosensory retina could explain this finding $[28,29]$. The median age 
in this study was $51 \pm 14$ years, and only one eye with chronic residual SRF was reported (Figure2 B-1 and B2); consequently, this variable was not considered as a cause of poor visual results.

In this retrospective multicenter study using SDOCT, we documented multiple structural alterations, such as diffuse thinning of the neurosensory macula (Figure1 E-2 image; Figure6 B-3 image), morphological alterations in the foveal contour (Figure6 A-1-A-3 images), a significant decrease in the mean CSFT, and ellipsoid band and ELM line reflectivity discontinuities (Figure2 C-1 image; Figure7 C-2 and C-3) in all three groups; a statistically significant predominance of these alterations was observed in the peeling group (Table 2). However, in this study, in the buckling group, the best functional results were significantly associated $(p<0.05)$ with the following variables: the presence or absence of significant postoperative ERM proliferation, RRD recurrence rate (Table 2), eventual ERM surgery, the presence or absence of submacular blood, and the event of ERM proliferation surgery; in the vitrectomy groups, the best functional results were observed in the presence of an intact or untouched ILM and absence of postoperative ERM proliferation at the end of follow-up (Table 2); evidently, prospective and multicenter studies are required to evaluate the SD-OCT findings recovered at serial and longitudinal follow-up in these patients, correlate these findings with visual recovery and final postoperative BCVA, and determine the role of the surgical removal of the ILM in macular and visual function.

Additional statistical analysis of the buckling group for the final postoperative BCVA after ERM surgery did not allow us to find any functional or categorical variables that were significantly associated with it; the GLM showed that postoperative BCVA was statistically significantly dependent on the following variables: postoperative ERM proliferation, which increased the postoperative BCVA by 0.68 in logMAR units, and retinal entrapment, which decreased the postoperative BCVA by 0.21 in logMAR units. The GLM also showed that the final postoperative BCVA after ERM surgery was statistically significantly dependent on the following variables: postoperative BCVA in logMAR units, preoperative BCVA in logMAR units, and retinal perforation, which increased the postoperative BCVA after ERM surgery by 0.15 in logMAR units.

We found a recurrence RRD rate of $1.82 \%$ in the non-peeling group, $24.0 \%$ in the peeling group, and $8.80 \%$ in the buckle group (Table 2). A recently published meta-analysis[30] reported a recurrence RRD rate between $28 \%$ and $21 \%$ after scleral buckle and primary vitrectomy, respectively, and Deiss et al.[31] reported a recurrence RRD rate of $25.55 \%$ after vitrectomy with ILM peeling in the treatment of primary macula involving RRD. Although in our report the recurrences were identified earlier in the buckle group comparatively with the vitrectomy group and consequently resolved timely, in the statistical analysis, this particular variable was not significant but relevant from a clinical point of view; in connection with this, we observed a high rate of recurrence of detachment in the peeling group (24.0\%) and speculated that perhaps the ILM removal maneuvers were intimately associated with the risk of producing tiny subclinical iatrogenic rhegmatogenous lesions, therefore significantly raising the incidence of somewhat late recurrences that went unnoticed and became apparent once the gastamponade disappeared.

Several reports have indicated poor functional results in eyes with non-complicated macula-off RRD managed with primary vitrectomy and ILM removal; it is well known that the involvement of the macula affects recovery; thus, we investigated what type of damage to the photoreceptors or external layers of the macula could be detected to explain the unfavorable recovery, especially in the peeling group without reaching plausible conclusions. We must recognize, however, that the possible additional mechanisms by which the removal of the ILM could cause a lack of functional recovery are still unclear, and additional prospective and multicenter studies are required[32], as mentioned above. We consider that the only indication for ILM removal in the management of a non-complicated macula-off RRD is to relieve or prevent postoperative macular traction caused by the presence of a welldocumented pre- or trans-operative ERM proliferation; therefore, a non-complicated macula-off RRD should be managed with vitrectomy and macular surgery involving the removal of ERM-ILM complex and additional scleral buckling performed at the surgeon's discretion. When the ILM was removed, the incidence of ERM was $0.003 \%$ [19] to $2.0 \%$ (Table 2) and ranged from $21.5 \%$ to $58 \%$ when the ILM was not removed. In case a preoperative ERM is concomitant with a noncomplicated macula-off RRD, a 3-port plana vitrectomy with concomitant en-bloc removal of the ERM-ILM membranes complex or ERM proliferation and ILM twostep (double-staining technique) peeling surgical removal should be considered as the first surgical approach. A prophylactic approach to prevent the formation of ERM proliferation over the macula is not currently justified in our experience and should be reserved for complicated cases. In our report, only one patient with significant ERM proliferation in the peeling group was detected (2.0\% incidence), probably due to an incomplete or failed ILM removal technique (Table 2). Some authors[30] have reported that the duration of RRD prior to primary vitrectomy is not a significant risk factor for postoperative BCVA; this variable was analyzed and compared across groups; however, one of the classic variables that best correlates with the final postoperative BCVA is precisely the shortest time that photoreceptors remain separated from the RPE. Herein, 
we found a strong positive correlation between the final BCVA and the mean time period of macular detachment before surgery (3.6 \pm 2.5 weeks in the buckle group and $4.4 \pm 2.6$ weeks in the vitrectomy group), which was considered similar in the groups studied but rather a long period with the macula detached. This factor possibly contributed to the poor functional and structural results, and together with the removal of ILM, may have contributed to the poorer functional results in the peeling group. The same authors reported a greater subfoveal thickness and lower final vision, which should be considered very cautiously since the thickness is also related to the patient's age, and choroidal structure deterioration and photoreceptor loss with aging could explain this finding[33]. We did not find any statistically significant association of the mean CSFT across groups (Table 2). We also did not find any significant association between the hypothetical predictive factors for ERM proliferation, such as age, sex, encircling buckle, transoperative use of perfluorocarbon liquids, and the postoperative presence of ERM, as reported by Schwartz et al.[22] and Schmidt et al.[34] However, the use of cryotherapy, external drainage complications such as retinal perforation, through and through SRF drainage complication phenomenon, vitreoretinal entrapment, subretinal bleeding, and the time of macular involvement before surgery showed consistent statistically significant values $(p<0.05)$ in the logistic regression analysis and were considered good surgical predictors for the final visual acuity (Additional Table S6A, S6B, and S6C).

This study has several strengths, such as the multicenter design and the long-term structural and multimodal functional analyses and complication analyses. However, it also has several limitations, mainly pertaining to its retrospective nature and limited size; accordingly, real-life conclusions cannot be obtained based on a few cases. but this report could be a stimulus for the elaboration of prospective and multicentric studies in relation to this pathology and its consequences and complications.

\section{Conclusions}

In summary, our findings suggest that noncomplicated macula-off RRD should be treated as soon as possible to minimize photoreceptor and RPE damage by involutional changes due to the loss of mechanical, biochemical, and nutritional contact between the photoreceptors and RPE. At present, we cannot determine whether the functional alterations were due to the mean exposure time in weeks of the photoreceptors to the SRF $(4.14 \pm 2.53$ for the general group) or whether they were secondary to possible mechanisms at the cellular level related to the removal of the ILM. Successful early macular anatomical reattachment could only result in subclinical damage, but if the detachment time of the macula is prolonged, significant functional sequelae were observed, as seen in the multimodal functional postoperative eye evaluation in this study.

In conclusion, based on the analyses of our results, as well as those of other authors, we concluded that the peeling of the ILM in non-complicated maculaoff RRD cases caused a reduction in glial cell proliferation by inhibiting the scarring process. Consequently, hopefully, our study might contribute with the findings of serious consequences in the structure and especially in the macular function of the eyes, as demonstrated by the analysis of the final vision, where the worst functional results in logMAR units, mfERG, and microperimetry evaluation are seen in the peeling group, although our results are compatible with those of other authors, we can conclusively state that removing the MLI with the main objective of avoiding postoperative or secondary macular ERM proliferation is not justified due to the high rate of potential complications and poor final visual results demonstrated in this study. No ERM proliferations developed in the peeling group; however, significant functional and structural differences among the buckle, peeling, and non-peeling groups were assessed using the mfERG, MRS, FRS, and en-face SDOCT findings of the peeled area, and the alterations found or the lack of recovery in the postoperative SDOCT biomarkers should raise deep concerns regarding the use of this technique in non-complicated cases if the only beneficial outcome is to avoid the development of ERM proliferation. If ERM proliferation does occur, it can be managed later, only if they are symptomatic or show significant structural and functional alterations in the macula as mentioned before. Further prospective randomized clinical trials are needed to better establish the role of ILM removal and determine the most appropriate surgical procedures to reduce the incidence of postoperative ERM proliferation. Although the number and complexity of major complications were significantly lower in the vitrectomy group compared to the buckle group, the multidisciplinary postoperative evaluation at long-term follow-up yielded a microstructurally and functionally abnormal macula in the three groups but predominantly in the peeling group $(p<0.05)$. Scleral buckling techniques still have a role in retinal detachment repair, and it remains an important skill for a retinal surgeon, but we need to refine the technique and reduce the risk factors that might raise the incidence of postoperative ERM, mainly the use of cryotherapy and complications related to transscleral drainage of SRF, as we describe in this report.

Sequential and serial postoperative structural and functional multimodal imaging techniques for the diagnosis and follow-up of retinal disorders are continuously being developed not only to offer more precise clinical diagnostic and prognostic insights but also to quantify the visual impact. The anatomical and 
functional results of this comparative, retrospective multicentric, long-term, one-surgeon study indicated significant visual damage at the clinical level when a non-complicated macula-off RRD is associated with primary or secondary postoperative ERM proliferation and must be resolved by performing vitrectomy complemented with ERM-ILM complex membranes removal techniques as described in this report.

\section{List of abbreviations}

ASR: additional surgery rate; BBG: Brilliant Blue G; BCVA: best-corrected visual acuity; CSFT: central subfoveal thickness; DONFL: dissociated optic nerve fiber layer; ELM, external limiting membrane; ERM: epiretinal membrane; FRS: foveal retinal sensitivity; ILM: internal limiting membrane; logMAR: logarithm of the minimum angle of resolution; mfERG: multifocal electroretinography; MRS: macular retinal sensitivity; OCT: optical coherence tomography; PFCL: perfluorocarbon liquid; PVR: proliferative vitreoretinopathy; RD: retinal detachment; RPE: retinal pigment epithelium; RRD: rhegmatogenous retinal detachment; SD-OCT: spectral-domain optical coherence tomography; SS: swept source

\section{Declarations}

Ethics approval and consent to participate: This retrospective study adhered to the tenets of the Declaration of Helsinki, received full ethical approval from the Research Ethics Committees, and was approved by the Institutional Review Committees and the Teaching Departments of the three institutions enrolled (no reference number is provided for retrospective studies by these institutions). Written informed consent in accordance with the institutional guidelines was obtained from all the patients.

Consent for publication: Each patient included in this report has given their written consent to be operated on and also their written consent for the publication of this report.

Availability of data and materials: Photos, composite figures, and laboratory studies supporting the findings of this study may be released upon written application to the Photographic, Psychophysics laboratory and Clinical Archives department at Instituto de Oftalmología Fundacíon Conde de Valenciana (Non-profit Organization), Chimalpopoca 14, Colonia Obrera, Mexico City 06800, Mexico and from the corresponding author upon request.

Competing interests; The authors declare that they have no competing interests.

Funding: No funding or grant support was received for this study.

Authors' contributions: MAQR, original idea, writing the manuscript, dataset interpretation, statistical analysis interpretation, final revision, and conclusions; EAQG, figures artwork, statistics, tables, and graphics; JGMN, statistics, tables, and graphics; FEC, photographic material compilation; JEAV, photographic material compilation; JHKL, photographic material compilation; ANJ, statistic correlation and final revision; MM, final revision and statistical analysis; VLG, final revision, statistical analysis, artwork and figure formatting, and figure text editing; FGW, final revision.

Acknowledgments: We express our deep appreciation to the technical staff of the three participant institutions, Retina Service at the Instituto de Oftalmologia, Fundacion Conde de Valenciana, Mexico City, Mexico; Retina specialist at the American British Cowdray hospital; and Retina service at the Hospital Juarez, Mexico.

\section{References Références Referencias}

1. Aylward GW, Orr G, Schwartz SD, Leaver PK. Prospective, randomised, controlled trial comparing suture needle drainage and argon laser drainage of subretinal fluid. Br J Ophthalmol. 1995; 79:724-7.

2. Chronopoulos A, Schutz JS, Varga Z, Souteyrand G, Thumann G. Complications of encircling bandsprevention and management. $J$ Clin Exp Ophthalmol. 2015; 6:2.

3. La Heij EC, Derhaag PFJM, Hendrikse F. Results of scleral buckling operations in primary rhegmatogenous retinal detachment. Doc Ophthalmol. 2000; 100:17-25.

4. Brown $P$, Chignell $A H$. Accidental drainage of subretinal fluid. Br J Ophthalmol. 1982; 66:625-6.

5. Stone TW, Han P. ASRS 2019. Preferences and trends membership survey. Chicago: American Society of Retina Specialists; 2019.

6. Halfter W, Willem M, Mayer U. Basement membrane-dependent survival of retinal ganglion cells. Inves Ophthalmol Vis Sci. 2005; 46:1000-9.

7. Almony A, Nudleman E, Shah GK, Blinder KJ, Eliott DB, Mittra RA, et al. Techniques, rationale, and outcomes of internal limiting membrane peeling. Retina. 2012; 32:877-91.

8. Hejsek L, Dusová J, Stepanov A, Rozsíval P. Internal limiting membrane peeling as prophylaxis of epimacular membrane formation in eyes undergoing vitrectomy for rhegmatogenous retinal detachement. Cesk Slov Oftalmol. 2014; 70:98-101.

9. Odrobina D, Bednarski M, Cisiecki S, Michalewska Z, Kuhn F, Nawrocki J. Internal limiting membrane peeling as prophylaxis of macular pucker formation in eyes undergoing retinectomy for severe proliferative vitreoretinopathy. Retina. 2012; 32: 226-31.

10. Hisatomi T, Tachibana T, Notomi $S$, Koyanagi $Y$, Murakami $Y$, Takeda A, et al. Internal limiting membrane peeling-dependent retinal structural 
changes after vitrectomy in rhegmatogenous retinal detachment. Retina. 2018; 38:471-9.

11. Akiyama K, Fujinami K, Watanabe K, Tsunoda K, Noda T. Internal limiting membrane peeling to prevent post-vitrectomy epiretinal membrane development in retinal detachment. Am J Ophthalmol. 2016; 171:1-10.

12. Nam KY, Kim JY. Effect of internal limiting membrane peeling on the development of epiretinal membrane after pars plana vitrectomy for primary rhegmatogenous retinal detachment. Retina. 2015; 35:880-5.

13. Venables WN, Ripley BD. Modern Applied Statistics with S. 4th ed. New York: Springer; 2002.

14. Tabandeh H, Khachaturov A, Rezaei KA, Boyer DS. Supplemental scleral buckle in the era of small incision vitrectomy and wide-angle viewing systems. Retina. 2020; 40:1894-9.

15. Tan HS, Mura M, Oberstein SY, de Smet MD. Primary retinectomy in proliferative vitreoretinopathy. Am J Ophthalmol. 2010; 149:447-52.

16. Schneider EW, Geraets RL, Johnson MW. Pars plana vitrectomy without adjuvant procedures for repair of primary rhegmatogenous retinal detachment. Retina. 2012; 32:213-9.

17. Tabandeh H, London NJS, Boyer DS, Flynn HW Jr. Outcomes of small-gauge vitreoretinal surgery without scleral-depressed shaving of the vitreous base in the era of wide-angle viewing systems. $\mathrm{Br} J$ Ophthalmol. 2019; 103:1765-8.

18. Bovey EH, Uffer S, Achache F. Surgery for epimacular membrane: impact of retinal internal limiting membrane removal on functional outcome. Retina. 2004; 24:728-35.

19. Eissa MGAM, Abdelhakim MASE, Macky TA, Khafagy MM, Mortada HA. Functional and structural outcomes of ILM peeling in uncomplicated maculaoff RRD using microperimetry \& en-face OCT. Graefes Arch Clin Exp Ophthalmol. 2018; 256: 249-57.

20. Garweg JG, Deiss M, Pfister IB, Gerhardt C. Impact of inner limiting membrane peeling on visual recovery after vitrectomy for primary rhegmatogenous retinal detachment involving the fovea. Retina. 2019; 39:853-9.

21. Tari SR, Vidne-Hay O, Greenstein VC, Barile GR, Hood DC, Chang S. Functional and structural measurements for the assessment of internal limiting membrane peeling in idiopathic macular pucker. Retina. 2007; 27: 567-72.

22. Schwartz SG, Flynn HW. Primary retinal detachment: scleral buckle or pars plana vitrectomy? Curr Opin Ophthalmol.2006; 17: 245-50.

23. Abdullah ME, Moharram HEM, Abdelhalim AS, Mourad KM, Abdelkader MF. Evaluation of primary internal limiting membrane peeling in cases with rhegmatogenous retinal detachment. Int journal of retina and vitreous. 2020; 6:1-8.

24. Haritoglou C, Gass CA, Schaumberger M, Ehrt O, Gandorfer A, Kampik A. Macular changes after peeling of the internal limiting membrane in macular hole surgery. Am J Ophthalmol. 2001; 132: 363-8.

25. Alkabes M, Salinas C, Vitale L, Burés-Jelstrup A, Nucci P, Mateo C. En face optical coherence tomography of inner retinal defects after internal limiting membrane peeling for idiopathic macular hole. Invest Ophthalmol Vis Sci. 2011; 52:8349-55.

26. Spaide RF. "Dissociated optic nerve fiber layer appearance" after internal limiting membrane removal is inner retinal dimpling. Retina. 2012; 32 : 1719-26.

27. Schuman RG, Gandorfer A, Priglinger SG, Kampik A, Haritoglou C. Vital dyes for macular surgery: a comparative electron microscopy study of the ILM. Retina. 2009; 29:669-76.

28. Balducci N, Morara M, Veronese C, Torrazza C, Pichi F, Ciardella AP. Retinal nerve fiber layer thickness modification after internal limiting membrane peeling. Retina. 2014; 34:655-63.

29. Chen H, Lukas TJ, Du N, Suyeoka G, Neufeld AH. Dysfunction of the retinal pigment epithelium with age: increased iron decreases phagocytosis and lysosomal activity. Invest Ophthalmol Vis Sci. 2009; 50:1895-902.

30. Znaor L, Medic A, Binder S, Vucinovic A, Marin Lovric J, Puljak L. Pars plana vitrectomy versus scleral buckling for repairing simple rhegmatogenous retinal detachments. Cochrane Database Syst Rev. 2019; 3:CD009562.

31. Deiss M, Kaya C, Pfister IB, Garweg JG.Impact of vitreal tamponade on functional outcomes in vitrectomy with ILM peeling in primary maculainvolving retinal detachment: a retrospective analysisClin Ophthalmol. 2020; 14:4493-500.

32. Ripandelli G, Scarinci F, Piaggi P, Guidi G, Pileri M, Cupo $G$, et al. Macular pucker: to peel or not to peel the internal limiting membrane? A microperimetric response. Retina. 2015; 35:498-507.

33. Fitzgerald ME, Tolley E, Frase S, Zagvazdin Y. Functional and morphological assessment of agerelated changes in the choroid and outer retina in pigeons. Vis Neurosci. 2001; 18:299-317.

34. Schmidt I, Plange $N$, Rößler $G$, Schellhase $H$, Koutsonas A, Walter $P$, et al.Long-term clinical results of vitrectomy and scleral buckling in treatment of rhegmatogenous retinal detachment. Scientific Word Journal. 2019; 2019:5416806. 
Table S1: Shapiro-Wilk normality test results.

\begin{tabular}{|c|c|c|c|}
\hline & Object & W & p value \\
\hline 1 & Age & 0.954 & 0.001 \\
\hline 2 & Preoperative BCVA (logMAR) & 0.930 & 0.001 \\
\hline 3 & Postoperative BCVA (logMAR) & 0.790 & 0.001 \\
\hline 4 & ERM Detection (weeks) & 0.961 & 0.319 \\
\hline 5 & BCVA after ERM surgery (logMAR) & 0.951 & 0.211 \\
\hline 6 & CSFT (microns) & 0.888 & 0.001 \\
\hline 7 & Follow-up period (months) & 0.959 & 0.001 \\
\hline
\end{tabular}

The variables that do not follow a normal distribution are in bold writing. $(p<0.05)$

W (Shapiro-Wilk normality test): BCVA: best corrected visual acuity; CSFT: central subfoveal thickness

Table S2: Descriptive statistics for the numeric variables.

\begin{tabular}{|c|c|c|c|c|c|c|}
\hline & Object & Mean & Min & Max & $\begin{array}{c}\text { Standard } \\
\text { Deviation }\end{array}$ & $\begin{array}{c}\text { Length of Sample } \\
(\mathrm{n}=125)\end{array}$ \\
\hline 1 & Age (years) & 44.34 & 18.00 & 76.00 & 15.94 & 125 \\
\hline 2 & Preoperative macula-off (weeks) & 3.60 & 1.00 & 12.00 & 2.47 & 125 \\
\hline 3 & Preoperative BCVA (logMAR) & 1.03 & 0.48 & 1.60 & 0.28 & 125 \\
\hline 4 & Postoperative BCVA (logMAR) & 0.40 & 0.10 & 1.30 & 0.33 & 125 \\
\hline 5 & ERM detection (weeks) & 11.93 & 5.00 & 22.00 & 4.59 & 125 \\
\hline 6 & BCVA after ERM surgery(logMAR) & 0.43 & 0.18 & 0.70 & 0.14 & 125 \\
\hline 7 & CSFT (microns) & 243.57 & 32.00 & 402.00 & 41.95 & 125 \\
\hline 8 & Follow-up period (months) & 26.11 & 2.00 & 73.00 & 13.42 & 125 \\
\hline
\end{tabular}

Non-parametric Mann-Whitney U-test. min: minimum; max: maximum; BCVA: best corrected visual acuity; ERM: epiretinal membrane; CSFT: central subfoveal thickness

Table S3: Summarized statistics for the categorical variables.

\begin{tabular}{|c|c|c|c|}
\hline Variable & Value & $\mathrm{n}$ & Freq \\
\hline \multirow{2}{*}{ Sex } & Female & 75 & 0.6 \\
\cline { 2 - 4 } & Male & 50 & 0.4 \\
\cline { 2 - 4 } & Left & 59 & 0.472 \\
\hline \multirow{3}{*}{ Eye } & Right & 66 & 0.528 \\
\hline \multirow{3}{*}{ Preop BCVA } & Phakic & 98 & 0.784 \\
\cline { 2 - 4 } & Pseudophakic & 27 & 0.216 \\
\cline { 2 - 4 } & $20 / 100$ & 26 & 0.208 \\
\cline { 2 - 4 } & $20 / 160$ & 14 & 0.112 \\
\cline { 2 - 4 } & $20 / 200$ & 12 & 0.28 \\
\cline { 2 - 4 } & $20 / 300$ & 21 & 0.096 \\
\cline { 2 - 4 } & $20 / 400$ & 1 & 0.168 \\
\cline { 2 - 4 } & $20 / 60$ & 3 & 0.008 \\
\cline { 2 - 4 } & $20 / 70$ & 11 & 0.016 \\
\cline { 2 - 4 } & $20 / 80$ & & \\
\hline
\end{tabular}




\begin{tabular}{|c|c|c|c|}
\hline \multirow[t]{13}{*}{ Postop BCVA } & $20 / 100$ & 11 & 0.088 \\
\hline & $20 / 120$ & 1 & 0.008 \\
\hline & $20 / 160$ & 1 & 0.008 \\
\hline & $20 / 200$ & 4 & 0.032 \\
\hline & $20 / 25$ & 16 & 0.128 \\
\hline & $20 / 30$ & 35 & 0.28 \\
\hline & $20 / 300$ & 3 & 0.024 \\
\hline & $20 / 40$ & 31 & 0.248 \\
\hline & $20 / 400$ & 6 & 0.048 \\
\hline & $20 / 50$ & 4 & 0.032 \\
\hline & $20 / 60$ & 8 & 0.064 \\
\hline & $20 / 70$ & 1 & 0.008 \\
\hline & $20 / 80$ & 4 & 0.032 \\
\hline \multirow[t]{2}{*}{ Redetachment } & No & 114 & 0.912 \\
\hline & Yes & 11 & 0.088 \\
\hline \multirow[t]{6}{*}{ Additional Surgery } & & 114 & 0.912 \\
\hline & BUCKLE REVISION & 4 & 0.032 \\
\hline & PHAKO-VITRECTOMY & 3 & 0.024 \\
\hline & $\begin{array}{l}\text { PHAKO-VITRECTOMY-ERM } \\
\text { PEELING }\end{array}$ & 1 & 0.008 \\
\hline & VITRECTOMY & 2 & 0.016 \\
\hline & VITRECTOMY-ERM PEELING & 1 & 0.008 \\
\hline \multirow[t]{2}{*}{ Postop ERM proliferations } & No & 96 & 0.768 \\
\hline & Yes & 29 & 0.232 \\
\hline \multirow[t]{2}{*}{ ERM Surgery } & No & 98 & 0.784 \\
\hline & Yes & 27 & 0.216 \\
\hline \multirow[t]{8}{*}{ BCVA after ERM surgery } & & 97 & 0.776 \\
\hline & $20 / 100$ & 2 & 0.016 \\
\hline & $20 / 30$ & 2 & 0.016 \\
\hline & $20 / 40$ & 7 & 0.056 \\
\hline & $20 / 50$ & 6 & 0.048 \\
\hline & $20 / 60$ & 4 & 0.032 \\
\hline & $20 / 70$ & 4 & 0.032 \\
\hline & $20 / 80$ & 3 & 0.024 \\
\hline \multirow[t]{2}{*}{ Retinal perforation } & No & 118 & 0.944 \\
\hline & Yes & 7 & 0.056 \\
\hline
\end{tabular}



Retrospective, Long-Term, Multicenter CASe Series Report

\begin{tabular}{|c|c|c|c|}
\hline \multirow[t]{2}{*}{ Submacular blood } & No & 120 & 0.96 \\
\hline & Yes & 5 & 0.04 \\
\hline \multirow{2}{*}{$\begin{array}{c}\text { Through and through scleral } \\
\text { drainage complication } \\
\text { phenomenon }\end{array}$} & No & 117 & 0.936 \\
\hline & Yes & 8 & 0.064 \\
\hline \multirow[t]{2}{*}{ Retinal entrapment } & No & 122 & 0.976 \\
\hline & Yes & 3 & 0.024 \\
\hline \multirow[t]{3}{*}{ Foveal contour OCT alterations } & Normal & 14 & 0.112 \\
\hline & Abnormal & 19 & 0.152 \\
\hline & Normal & 92 & 0.736 \\
\hline \multirow[t]{3}{*}{ Ellipsoid band OCT alterations } & & 14 & 0.112 \\
\hline & Disrupted & 25 & 0.2 \\
\hline & Normal & 86 & 0.688 \\
\hline \multirow[t]{3}{*}{ DONFL OCT defects } & & 14 & 0.112 \\
\hline & Not Present & 80 & 0.64 \\
\hline & Present & 31 & 0.248 \\
\hline \multirow[t]{3}{*}{ ELM line OCT alterations } & & 15 & 0.12 \\
\hline & Abnormal & 24 & 0.192 \\
\hline & Normal & 86 & 0.688 \\
\hline \multirow[t]{3}{*}{ mfERGregistration } & & 26 & 0.208 \\
\hline & Abnormal & 54 & 0.432 \\
\hline & Normal & 45 & 0.36 \\
\hline \multirow[t]{3}{*}{ Microperimetry results } & & 18 & 0.144 \\
\hline & Abnormal & 51 & 0.408 \\
\hline & Normal & 56 & 0.448 \\
\hline
\end{tabular}

Fisher's exact test. freq: frequency; preop: preoperative: postop: postoperative: BCVA: best corrected visual acuity; CSFT: central subfoveal thickness; ERM: epiretinal membrane; DONFL: diffuse optic nerve fiber layer; ELM: external limiting membrane: mfERG: multifocal electroretinography

Table S4: Correlations among the numeric variables in the Buckle group (sample size $\mathrm{N}=125$ eyes)

\begin{tabular}{|c|c|c|c|c|c|c|c|c|}
\hline & Age & $\begin{array}{l}\text { Preoperative } \\
\text { macula-off } \\
\text { (weeks) }\end{array}$ & $\begin{array}{c}\text { Preoperative } \\
\text { BCVA } \\
\text { (logMAR) }\end{array}$ & $\begin{array}{c}\text { Postoperative } \\
\text { BCVA } \\
\text { (logMAR) }\end{array}$ & $\begin{array}{c}\text { ERM } \\
\text { Detection } \\
\text { (weeks) }\end{array}$ & \begin{tabular}{|c|} 
BCVA \\
After ERM \\
Surgery \\
(logMAR) \\
\end{tabular} & $\begin{array}{c}\mathrm{CSFT} \\
\text { (microns) }\end{array}$ & $\begin{array}{l}\text { Follow-up } \\
\text { Period } \\
\text { (months) }\end{array}$ \\
\hline Age & $1(p=N A)$ & & & & & & & \\
\hline $\begin{array}{c}\text { Preoperative macula-off } \\
\text { (weeks) }\end{array}$ & $\begin{array}{c}0.12 \\
(p=0.17)\end{array}$ & $1(p=N A)$ & & & & & & \\
\hline $\begin{array}{c}\text { Preoperative BCVA } \\
\text { (IogMAR) }\end{array}$ & $\begin{array}{c}0.01 \\
(p=0.88)\end{array}$ & $\begin{array}{c}0.04 \\
(p=0.63)\end{array}$ & $1(p=N A)$ & & & & & \\
\hline $\begin{array}{c}\text { Postoperative BCVA } \\
\text { (logMAR) }\end{array}$ & $\begin{array}{c}-0.06 \\
(p=0.48)\end{array}$ & $\begin{array}{c}-0.02 \\
(p=0.78)\end{array}$ & $\begin{array}{c}0.02 \\
(p=0.85)\end{array}$ & $1(p=N A)$ & & & & \\
\hline ERM detection (weeks) & $\begin{array}{c}-0.27 \\
(p=0.15)\end{array}$ & $\begin{array}{c}-0.19 \\
(p=0.31)\end{array}$ & $\begin{array}{c}-0.19 \\
(p=0.31)\end{array}$ & $0.06(p=0.74)$ & $1(p=N A)$ & & & \\
\hline $\begin{array}{c}\text { BCVA after ERM surgery } \\
\text { (logMAR) }\end{array}$ & $\begin{array}{c}0.05 \\
(p=0.79) \\
\end{array}$ & $\begin{array}{c}-0.21 \\
(p=0.28)\end{array}$ & $\begin{array}{c}0.26 \\
(p=0.17)\end{array}$ & $0.57(p=0.00)$ & $\begin{array}{c}0.04 \\
(p=0.82)\end{array}$ & $1(p=N A)$ & & \\
\hline
\end{tabular}




\begin{tabular}{|c|c|c|c|c|c|c|c|c|}
\hline CSFT (microns) & $\begin{array}{c}0.01 \\
(p=0.9)\end{array}$ & $\begin{array}{c}0.01 \\
(p=0.93)\end{array}$ & $\begin{array}{c}0.09 \\
(p=0.37)\end{array}$ & $0.13(p=0.17)$ & $\begin{array}{c}0.04 \\
(p=0.85)\end{array}$ & $\begin{array}{c}-0.06 \\
(p=0.78)\end{array}$ & $1(p=N A)$ \\
\hline $\begin{array}{c}\text { Follow-up period } \\
\text { (months) }\end{array}$ & $\begin{array}{c}-0.17 \\
(p=0.06)\end{array}$ & $\begin{array}{c}-0.17 \\
(p=0.06)\end{array}$ & $\begin{array}{c}0.03 \\
(p=0.71)\end{array}$ & $-0.2(p=0.03)$ & $\begin{array}{c}0.08 \\
(p=0.68)\end{array}$ & $\begin{array}{c}-0.05 \\
(p=0.79)\end{array}$ & $\begin{array}{c}0.12 \\
(p=0.2)\end{array}$ & $1(p=N A)$ \\
\hline
\end{tabular}

Spearman's rank correlation coefficient test. The significant correlations are in bold text. BCVA: best corrected visual acuity; ERM: epiretinal membrane; CSFT: central subfoveal thickness; NA: not applicable

Table S5: A) PreopBCVA, B) Postop BCVA C) BCVA after ERM surgery in the Buckle group ( $\mathrm{N}=125$ eyes)

\begin{tabular}{|l|c|c|}
\hline A. preoperative BCVA (logMAR) Mann-Whitney U tests results & & \\
\hline Object & $\mathrm{U}$ & p value \\
\hline Age & 7875 & 0.001 \\
\hline Preoperative macula-off (weeks) & 7140 & 0.001 \\
\hline Postoperative BCVA (logMAR) & 201.5 & 0.001 \\
\hline ERM detection (weeks) & 465 & 0.001 \\
\hline BCVA after ERM surgery (logMAR) & 0 & 0.001 \\
\hline CSFT (microns) & 6105 & 0.001 \\
\hline Follow-up period (months) & 7875 & 0.001 \\
\hline B. postoperative BCVA (logMAR) Mann-Whitney U tests results & & \\
\hline Object & $\mathrm{U}$ & $\mathrm{p}$ value \\
\hline Age & 7875 & 0.001 \\
\hline Preoperative macula-off (weeks) & 7866 & 0.001 \\
\hline Preoperative BCVA (logMAR) & 7301.5 & 0.001 \\
\hline ERM detection (weeks) & 465 & 0.001 \\
\hline BCVA after ERM surgery (logMAR) & 0 & 0.001 \\
\hline CSFT (microns) & 6105 & 0.001 \\
\hline Follow-up period (months) & 7875 & 0.001 \\
\hline C. BCVA after ERM surgery (logMAR) Mann-Whitney U tests results & & \\
\hline Object & $\mathrm{U}$ & $\mathrm{p}$ value \\
\hline Age & 406 & 0.001 \\
\hline Preoperative macula-off (weeks) & 406 & 0.001 \\
\hline Preoperative BCVA (logMAR) & 378 & 0.001 \\
\hline Postoperative BCVA (logMAR) & 406 & 0.001 \\
\hline ERM detection (weeks) & 406 & 0.001 \\
\hline CSFT (microns) & 406 & 0.001 \\
\hline Follow-up period (months) & 406 & 0.001 \\
\hline
\end{tabular}

Mann-Whitney $U$ tests. The statistically significant variables $(p<0.05)$ are in bold text. $U$ testBCVA: best corrected visual acuity; ERM: epiretinal membrane; CSFT: central subfoveal thickness

Table S6A: Kruskal-Wallis results of the preoperative BCVA with the categorical variables in the Buckle group

\begin{tabular}{|c|c|c|c|c|c|c|}
\hline & Object & Kruskal-Wallis $\mathrm{x}^{2}$. & $\mathrm{df}$ & $\mathrm{p}$ value & Number of eyes & No. of NAs \\
\hline 1 & Male & 3.117 & 1 & 0.077 & 125 & 0 \\
\hline 2 & Eye & 2.132 & 1 & 0.144 & 125 & 0 \\
\hline 3 & Preoperative Lens Status & 0.130 & 1 & 0.718 & 125 & 0 \\
\hline 4 & Preoperative BCVA & 124.000 & 8 & 0.000 & 125 & 0 \\
\hline 5 & Postoperative BCVA & 5.342 & 12 & 0.946 & 125 & 0 \\
\hline 6 & Re-Detachment & 1.018 & 1 & 0.313 & 125 & 0 \\
\hline 7 & Additional surgery & 1.238 & 4 & 0.872 & 125 & 114 \\
\hline 8 & $\begin{array}{c}\text { Postoperative } \\
\text { ERM proliferations }\end{array}$ & 0.035 & 1 & 0.851 & 125 & 0 \\
\hline 9 & ERM surgery & 0.090 & 1 & 0.764 & 125 & 0 \\
\hline 10 & BCVA after ERM surgery & 4.763 & 6 & 0.575 & 125 & 97 \\
\hline
\end{tabular}




\begin{tabular}{|l|c|c|c|c|c|c|}
\hline 11 & Retinal perforation & 0.222 & 1 & 0.638 & 125 & 0 \\
\hline 12 & Submacular blood & 1.057 & 1 & 0.304 & 125 & 0 \\
\hline 13 & Through and through & 2.829 & 1 & 0.093 & 125 & 0 \\
\hline 14 & Retinal entrapment & 0.001 & 1 & 0.980 & 125 & 0 \\
\hline 15 & Foveal contour & 0.006 & 1 & 0.936 & 125 & 14 \\
\hline 16 & Ellipsoid & 0.236 & 1 & 0.627 & 125 & 14 \\
\hline 17 & DONFL & 1.581 & 1 & 0.209 & 125 & 14 \\
\hline 18 & ELM & 0.384 & 1 & 0.535 & 125 & 15 \\
\hline 19 & mfERG & 0.242 & 1 & 0.623 & 125 & 26 \\
\hline 20 & Microperimetry & 0.653 & 1 & 0.419 & 125 & 18 \\
\hline
\end{tabular}

Table S6B: Kruskal-Wallis results of the postoperative BCVA with the categorical variables

\begin{tabular}{|c|c|c|c|c|c|c|}
\hline & Object & Kruskal-Wallis $\mathrm{x}^{2}$. & df & $p$ value & Number of eyes & No. of NAs \\
\hline 1 & Male & 0.026 & 1 & 0.871 & 125 & 0 \\
\hline 2 & Eye & 0.047 & 1 & 0.828 & 125 & 0 \\
\hline 3 & Preoperative Lens Status & 0.234 & 1 & 0.629 & 125 & 0 \\
\hline 4 & Preoperative BCVA & 3.950 & 8 & 0.862 & 125 & 0 \\
\hline 5 & Postoperative BCVA & 124.000 & 12 & 0.000 & 125 & 0 \\
\hline 6 & Re-Detachment & 7.484 & 1 & 0.006 & 125 & 0 \\
\hline 7 & Additional surgery & 5.331 & 4 & 0.255 & 125 & 114 \\
\hline 8 & $\begin{array}{c}\text { Postoperative } \\
\text { ERMproliferations }\end{array}$ & 68.187 & 1 & 0.000 & 125 & 0 \\
\hline 9 & ERM surgery & 63.098 & 1 & 0.000 & 125 & 0 \\
\hline 10 & BCVA after ERM surgery & 13.048 & 6 & 0.042 & 125 & 97 \\
\hline 11 & Retinal perforation & 1.214 & 1 & 0.271 & 125 & 0 \\
\hline 12 & Submacular blood & 9.449 & 1 & 0.002 & 125 & 0 \\
\hline 13 & Through and Through & 0.357 & 1 & 0.550 & 125 & 0 \\
\hline 14 & Retinal Entrapment & 0.612 & 1 & 0.434 & 125 & 0 \\
\hline 15 & Foveal contour & 15.821 & 1 & 0.000 & 125 & 14 \\
\hline 16 & Ellipsoid & 3.479 & 1 & 0.062 & 125 & 14 \\
\hline 17 & DONFL & 18.677 & 1 & 0.000 & 125 & 14 \\
\hline 18 & ELM & 0.303 & 1 & 0.582 & 125 & 15 \\
\hline 19 & mfERG & 20.558 & 1 & 0.000 & 125 & 26 \\
\hline 20 & Microperimetry & 11.826 & 1 & 0.001 & 125 & 18 \\
\hline
\end{tabular}

Table S6C: Kruskal-Wallis results of the BCVA after ERM surgery with the categorical variables

\begin{tabular}{|c|c|c|c|c|c|c|}
\hline & Object & Kruskal-Wallis $\mathrm{x}^{2}$. & $\mathrm{df}$ & $\mathrm{p}$ value & Number of eyes & No. of NAs \\
\hline 1 & Male & 0.499 & 1 & 0.480 & 125 & 0 \\
\hline 2 & Eye & 0.967 & 1 & 0.325 & 125 & 0 \\
\hline 3 & Preoperative Lens Status & 1.070 & 1 & 0.301 & 125 & 0 \\
\hline 4 & Preoperative BCVA & 6.587 & 7 & 0.473 & 125 & 0 \\
\hline 5 & Postoperative BCVA & 11.572 & 6 & 0.072 & 125 & 0 \\
\hline 6 & Re-Detachment & 0.428 & 1 & 0.513 & 125 & 0 \\
\hline 7 & Additional surgery & 1.716 & 3 & 0.633 & 125 & 114 \\
\hline 8 & $\begin{array}{c}\text { Postoperative } \\
\text { ERM proliferations }\end{array}$ & 0.063 & 1 & 0.801 & 125 & 0 \\
\hline 9 & ERM surgery & 0.063 & 1 & 0.801 & 125 & 0 \\
\hline 10 & BCVA after ERM surgery & 27.000 & 6 & 0.000 & 125 & 97 \\
\hline 11 & Retinal perforation & 1.847 & 1 & 0.174 & 125 & 0 \\
\hline 12 & Submacular blood & 2.783 & 1 & 0.095 & 125 & 0 \\
\hline 13 & Through and Through & 1.144 & 1 & 0.285 & 125 & 0 \\
\hline
\end{tabular}




\begin{tabular}{|c|c|c|c|c|c|c|}
\hline 14 & Retinal entrapment & 0.776 & 1 & 0.378 & 125 & 0 \\
\hline 15 & Foveal contour & 0.178 & 1 & 0.673 & 125 & 14 \\
\hline 16 & Ellipsoid & 1.235 & 1 & 0.266 & 125 & 14 \\
\hline 17 & DONFL & 1.230 & 1 & 0.267 & 125 & 14 \\
\hline 18 & ELM & 0.138 & 1 & 0.710 & 125 & 15 \\
\hline 19 & mfERG & 0.115 & 1 & 0.734 & 125 & 26 \\
\hline 20 & Microperimetry & 1.033 & 1 & 0.310 & 125 & 18 \\
\hline
\end{tabular}

The statistically significant variables $(p<0.05)$ are in bold text.df: difference no: number; NA: not applicable; BCVA: best corrected visual acuity; ERM: epiretinal membrane; DONFL: diffuse optic nerve fiber layer; ELM: external limiting membrane: mfERG: multifocal electroretinography

Table S7: Generalized Linear Model results in the Buckle group ( $\mathrm{n}=125$ eyes)

\begin{tabular}{|c|c|c|c|c|c|}
\hline Preoperative BVCA & Estimate & Std. Error & t value & $\operatorname{Pr}(>|t|)$ & \\
\hline (Intercept) & 0.972 & 0.033 & 29.046 & $<2 \mathrm{e}-16$ & $\star \star * *$ \\
\hline Sex Male & 0.106 & 0.051 & 2.07 & 0.040 & * \\
\hline $\begin{array}{l}\text { Through and Throughscleral drainage } \\
\text { complication phenomenon }\end{array}$ & 0.164 & 0.102 & 1.605 & 0.111 & \\
\hline \multicolumn{6}{|l|}{ Generalized } \\
\hline Postoperative BCVA & Estimate & Std. Error & t value & $\operatorname{Pr}(>|t|)$ & \\
\hline (Intercept) & 0.218 & 0.056 & 3.854 & 0.001 & $\star \star \star$ \\
\hline Postop ERM proliferations & 0.676 & 0.035 & 19.055 & $<2 \mathrm{e}-16$ & 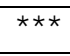 \\
\hline Retinal entrapment & -0.206 & 0.097 & -2.112 & 0.036 & $\star$ \\
\hline Preop BCVA logMAR & 0.029 & 0.052 & 0.567 & 0.572 & \\
\hline BCVA after ERM surgery & Estimate & Std. Error & t value & $\operatorname{Pr}(>|t|)$ & \\
\hline (Intercept) & -0.170 & 0.130 & -1.303 & 0.206 & \\
\hline Post BCVA logMAR & 0.323 & 0.080 & 4.003 & 0.001 & 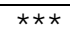 \\
\hline Preop BCVA logMAR & 0.194 & 0.072 & 2.694 & 0.013 & * \\
\hline Retinal perforation & 0.151 & 0.067 & 2.251 & 0.034 & * \\
\hline Age & 0.002 & 0.001 & 1.712 & 0.100 & \\
\hline Sex Male & -0.021 & 0.043 & -0.498 & 0.623 & \\
\hline
\end{tabular}

The statistically significant variables $(p<0.05)$ are in bold text and marked with *.

Pr: Probabilities using the $t$ distribution, gives the $p$-value for that $t$-test; BCVA: best corrected visual acuity; Postop: postoperative; Preop: preoperative: ERM: epiretinal membrane

Table S8: Shapiro-Wilk normality tests results in the Vitrectomy group ( $\mathrm{n}=105$ eyes).

\begin{tabular}{|c|c|c|}
\hline Object & W & P value \\
\hline Age (years) & 0.974 & 0.039 \\
\hline Macula-off (weeks) & 0.924 & 0.001 \\
\hline Preoperative BCVA (logMAR) & 0.923 & 0.001 \\
\hline Follow-up period (days) & 0.971 & 0.023 \\
\hline BCVA before ERM-ILM removal (logMAR) & 0.888 & 0.001 \\
\hline Final postoperative BCVA (logMAR) & 0.924 & 0.001 \\
\hline CSFT (microns) & 0.939 & 0.008 \\
\hline Follow-up period (months) & 0.970 & 0.023 \\
\hline
\end{tabular}

The variables that do not follow a normal distribution are in bold text ( $p<0.05)$. BCVA: best corrected visual acuity; ERM: epiretinal membrane; ILM: internal limiting membrane; CSFT: central subfoveal thickness 
Table S9: Descriptive statistics for the numeric variables in the Vitrectomy group

\begin{tabular}{|c|c|c|c|c|}
\hline Object & Mean & Min & Max & $\begin{array}{c}\text { Standard } \\
\text { Deviation }\end{array}$ \\
\hline Age (years) & 47.92 & 18.00 & 76.00 & 14.60 \\
\hline Macula-off (weeks) & 4.42 & 1.00 & 12.00 & 2.56 \\
\hline Preoperative BCVA (logMAR) & 1.06 & 0.54 & 1.60 & 0.27 \\
\hline Follow-up period (months) & 24.2 & 1.0 & 58.66 & 13.02 \\
\hline BCVA before ERM-ILM removal (logMAR) & 0.52 & 0.10 & 1.30 & 0.36 \\
\hline ERM detection (weeks) & 13.75 & 5.00 & 30.00 & 5.33 \\
\hline Final postoperative BCVA (logMAR) & 0.37 & 0.10 & 1.00 & 0.20 \\
\hline CSFT (microns) & 256.55 & 198.00 & 320.00 & 35.16 \\
\hline Follow-up period (months) & 23.42 & 1.00 & 57.00 & 12.98 \\
\hline
\end{tabular}

Wilcoxon rank sum test. Min: minimum; Max: maximum; BCVA: best corrected visual acuity; ERM: epiretinal membrane; ILM: internal limiting membrane; CSFT: central subfoveal thickness

Table S10: Summarized statistics for the categorical variables in the vitrectomy group (peeling and nonpeeling groups)

\begin{tabular}{|c|c|c|c|c|}
\hline Variable & Group & $\mathrm{n}$ & freq & $\%$ freq \\
\hline \multirow[t]{5}{*}{ Additional Surgery } & BUCKLE REVISION & 3 & 0.03 & $2.9 \%$ \\
\hline & No & 92 & 0.88 & $87.6 \%$ \\
\hline & PHAKO VITRECTOMY ERM PEELING & 1 & 0.01 & $1.0 \%$ \\
\hline & VITRECTOMY & 2 & 0.02 & $1.9 \%$ \\
\hline & VITRECTOMY REVISION & 7 & 0.07 & $6.7 \%$ \\
\hline \multirow[t]{3}{*}{ DONFL } & Absent & 34 & 0.32 & $32.4 \%$ \\
\hline & Present & 60 & 0.57 & $57.1 \%$ \\
\hline & NA & 11 & 0.10 & $10.5 \%$ \\
\hline \multirow[t]{2}{*}{ Ellipsoid } & Disrupted & 29 & 0.28 & $27.6 \%$ \\
\hline & Normal & 76 & 0.72 & $72.4 \%$ \\
\hline \multirow[t]{3}{*}{ ELM } & Disrupted & 27 & 0.26 & $25.7 \%$ \\
\hline & Normal & 74 & 0.70 & $70.5 \%$ \\
\hline & NA & 4 & 0.04 & $3.8 \%$ \\
\hline \multirow[t]{5}{*}{ ERM 2nd Surgery } & VIT and MACULA REVISION & 1 & 0.01 & $1.0 \%$ \\
\hline & VIT REVISION ERM-ILM REMOVAL & 45 & 0.43 & $42.9 \%$ \\
\hline & VIT REVISION ERM-ILM REMOVAL & 5 & 0.05 & $4.8 \%$ \\
\hline & VIT REVISION ERM-ILM REMOVAL & 1 & 0.01 & $1.0 \%$ \\
\hline & NA & 53 & 0.50 & $50.5 \%$ \\
\hline \multirow[t]{2}{*}{ Eye } & Left & 50 & 0.48 & $47.6 \%$ \\
\hline & Right & 55 & 0.52 & $52.4 \%$ \\
\hline \multirow[t]{2}{*}{ First Surgery } & BUCKLE & 27 & 0.26 & $25.7 \%$ \\
\hline & ONLY VITRECTOMY & 68 & 0.65 & $64.8 \%$ \\
\hline
\end{tabular}




\begin{tabular}{|c|c|c|c|c|}
\hline & VIT ERM-ILM REMOVAL & 10 & 0.10 & $9.5 \%$ \\
\hline \multirow[t]{3}{*}{ Foveal contour } & Abnormal & 24 & 0.23 & $22.9 \%$ \\
\hline & Normal & 77 & 0.73 & $73.3 \%$ \\
\hline & NA & 4 & 0.04 & $3.8 \%$ \\
\hline \multirow[t]{2}{*}{ Sex } & Female & 37 & 0.35 & $35.2 \%$ \\
\hline & Male & 68 & 0.65 & $64.8 \%$ \\
\hline \multirow[t]{3}{*}{ mfERG } & Abnormal & 43 & 0.41 & $41.0 \%$ \\
\hline & Normal & 30 & 0.29 & $28.6 \%$ \\
\hline & NA & 32 & 0.30 & $30.5 \%$ \\
\hline \multirow[t]{3}{*}{ Microperimetry } & Abnormal & 35 & 0.33 & $33.3 \%$ \\
\hline & Normal & 42 & 0.40 & $40.0 \%$ \\
\hline & NA & 28 & 0.27 & $26.7 \%$ \\
\hline \multirow{2}{*}{$\begin{array}{c}\text { Postoperative ERM } \\
\text { proliferations }\end{array}$} & No & 54 & 0.51 & $51.4 \%$ \\
\hline & Yes & 51 & 0.49 & $48.6 \%$ \\
\hline \multirow{2}{*}{$\begin{array}{c}\text { Preoperative ERM } \\
\text { proliferations }\end{array}$} & No & 55 & 0.52 & $52.4 \%$ \\
\hline & Yes & 50 & 0.48 & $47.6 \%$ \\
\hline \multirow[t]{2}{*}{ Preop Lens Status } & Phakic & 68 & 0.65 & $64.8 \%$ \\
\hline & Pseudophakic & 37 & 0.35 & $35.2 \%$ \\
\hline \multirow[t]{2}{*}{ Recurrent RRD } & No & 92 & 0.88 & $87.6 \%$ \\
\hline & Yes & 13 & 0.12 & $12.4 \%$ \\
\hline
\end{tabular}

Fisher's exact test. freq: frequency; ERM: epiretinal membrane; DONFL: diffuse optic nerve fiber layer; ELM: external limiting membrane; VIT: vitrectomy; ILM: internal limiting membrane; mfERG: multifocal electroretinography; RRD: rhegmatogenous retinal detachment

Table S11: Descriptive statistics with respect to the Vitrectomy (preoperative ERM proliferations) group (nonpeeling and peeling)

\begin{tabular}{|c|c|c|c|c|}
\hline Vitrectomy groups & Nonpeeling & peeling & $\mathrm{p}$ & \\
\hline & $(\mathrm{N}=55)$ & $(\mathrm{N}=50)$ & & \\
\hline & & & & \\
\hline Age & $50.455 \pm 13.52$ & $45.140 \pm 15.36$ & 0.054 & \\
\hline Sex & & & & \\
\hline - Female & & & & \\
\hline - Male & $19(34.545 \%)$ & $18(36.0 \%)$ & & \\
\hline & $36(65.455 \%)$ & $32(64.0 \%)$ & & \\
\hline Eye & & & & \\
\hline - Left & & & & \\
\hline - Right & $30(54.545 \%)$ & $25(50.0 \%)$ & & \\
\hline & & & & \\
\hline Preoperative Lens Status & & & & \\
\hline
\end{tabular}



Retrospective, Long-Term, Multicenter Case Series Report

\begin{tabular}{|c|c|c|c|c|}
\hline - Phakic & $31(56.364 \%)$ & $37(74.0 \%)$ & & \\
\hline - Pseudophakic & $24(43.636 \%)$ & $13(26.0 \%)$ & & \\
\hline Macula-off (weeks) & $4.527 \pm 2.403$ & $4.300 \pm 2.750$ & 0.425 & \\
\hline Preoperative BCVA (logMAR) & $1.036 \pm 0.258$ & $1.077 \pm 0.277$ & 0.386 & \\
\hline Follow-up period (days) & $768.6 \pm 373.01$ & $679.90 \pm 407.98$ & 0.131 & \\
\hline First Surgery & & & $0 * \star \star$ & \\
\hline - BUCKLE & $0(0.0 \%)$ & $27(54.0 \%)$ & & \\
\hline - ONLY VITRECTOMY & $55(100.000 \%)$ & $13(26.0 \%)$ & & \\
\hline - VIT ERM and ILM REMOVAL & $0(0.0 \%)$ & $10(20.0 \%)$ & & \\
\hline BCVA Before ERM-ILM removal (logMAR) & $0.297 \pm 0.23$ & $0.756 \pm 0.319$ & 0.001 & $* * *$ \\
\hline Recurrent RRD & & & 0.001 & $\star * \star$ \\
\hline$-\mathrm{No}$ & $54(98.182 \%)$ & $38(76.0 \%)$ & & \\
\hline - YES & $1(1.818 \%)$ & $12(24.0 \%)$ & & \\
\hline Additional Surgery & & & 0.004 & $* \star *$ \\
\hline - BUCKLE REVISION & $0(0.0 \%)$ & $3(6.0 \%)$ & & \\
\hline- No & $54(98.182 \%)$ & $38(76.0 \%)$ & & \\
\hline - PHAKO VITRECTOMY ERM PEELING & $0(0.0 \%)$ & $1(2.0 \%)$ & & \\
\hline - VITRECTOMY & $0(0.0 \%)$ & $2(4.0 \%)$ & & \\
\hline - VITRECTOMY REVISION & $1(1.818 \%)$ & $6(12.0 \%)$ & & \\
\hline ERM Detection (weeks) & $18.00 \pm 6.45$ & $12.575 \pm 4.385$ & 0.009 & *** \\
\hline ERM 2nd Surgery & & & 0 & $* \star *$ \\
\hline - VIT and MACULA REVISION & $0(0.0 \%)$ & $1(2.439 \%)$ & & \\
\hline - VIT REVISION ERM and ILM & $5(45.455 \%)$ & $40(97.561 \%)$ & & \\
\hline - VIT REVISION ERM and ILM REMOVAL & $5(45.455 \%)$ & $0(0.0 \%)$ & & \\
\hline - VIT REVISION ERM.ILM REMOVAL & $1(9.091 \%)$ & $0(0.0 \%)$ & & \\
\hline $\begin{array}{c}\text { Final Postoperative BCVA } \\
\text { (logMAR) }\end{array}$ & $0.280 \pm 0.192$ & $0.477 \pm 0.161$ & 0.001 & $* \star *$ \\
\hline CSFT (microns) & $266.71 \pm 32.75$ & $253.073 \pm 35.66$ & 0.173 & \\
\hline
\end{tabular}




\begin{tabular}{|c|c|c|c|c|}
\hline Foveal Contour & & & 0.002 & *** \\
\hline - Abnormal & $6(11.321 \%)$ & $18(37.5 \%)$ & & \\
\hline - Normal & $47(88.679 \%)$ & $30(62.5 \%)$ & & \\
\hline Ellipsoid integrity & & & 0.828 & \\
\hline - Disrupted & $16(29.091 \%)$ & $13(26.0 \%)$ & & \\
\hline - Normal & $39(70.909 \%)$ & $37(74.0 \%)$ & & \\
\hline DONFL defects & & & 0 & $\star \star \star ~$ \\
\hline - Absent & 39 (88.63\%) & $21(42.0 \%)$ & & \\
\hline - Present & $5(11.36 \%)$ & $29(58.0 \%)$ & & \\
\hline ELM line appearance & & & 0.654 & \\
\hline - Disrupted & $16(29.091 \%)$ & $11(23.913 \%)$ & & \\
\hline - Normal & $39(70.909 \%)$ & $35(76.087 \%)$ & & \\
\hline mfERG result & & & 0 & $* * *$ \\
\hline - Abnormal & $13(33.333 \%)$ & $30(88.235 \%)$ & & \\
\hline - Normal & $26(66.667 \%)$ & $4(11.765 \%)$ & & \\
\hline Microperimetry evaluation & & & 0 & 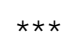 \\
\hline - Abnormal & $11(25.581 \%)$ & $24(70.588 \%)$ & & \\
\hline - Normal & $32(74.419 \%)$ & $10(29.412 \%)$ & & \\
\hline Follow-up period (months) & $24.80 \pm 12.34$ & $21.880 \pm 13.324$ & 0.133 & \\
\hline
\end{tabular}

The $p$-values ( $p$ ) are the results from the Wilcoxon rank sum test for the numerical variables and Fisher's Exact test for the categorical variables. The variables that showed a statistically significant difference $(p<0.05)$ among the groups of the peeling (preoperative ERM proliferation) are in boldtext and marked with *. sig: significance;BCVA: best corrected visual acuity; VIT: vitrectomy; ERM: epiretinal membrane; ILM: internal limiting membrane; RRD: rhegmatogenous retinal detachment; CSFT: central subfoveal thickness; DONFL: diffuse optic nerve fiber layer; ELM: external limiting membrane: mfERG: multifocal electroretinography.

Table S12: Correlations among the numeric variables in the Vitrectomy group (peeling and nonpeeling groups)

\begin{tabular}{|c|c|c|c|c|c|c|c|c|}
\hline & Age & $\begin{array}{l}\text { Preoperative } \\
\text { Macula-Off } \\
\text { (weeks) }\end{array}$ & $\begin{array}{c}\text { Preoperative } \\
\text { BCVA } \\
\text { (logMAR) }\end{array}$ & $\begin{array}{c}\text { BCVA } \\
\text { Before } \\
\text { ERM and } \\
\text { ILM } \\
\text { removal } \\
\text { (logMAR) }\end{array}$ & $\begin{array}{c}\text { ERM } \\
\text { Detection } \\
\text { (Weeks) }\end{array}$ & $\begin{array}{c}\text { Final } \\
\text { Postoperative } \\
\text { BCVA } \\
\text { (logMAR) }\end{array}$ & $\begin{array}{c}\text { CSFT } \\
\text { (microns) }\end{array}$ & $\begin{array}{l}\text { Follow- } \\
\text { up } \\
\text { period } \\
\text { (months) }\end{array}$ \\
\hline Age & 1 & & & & & & & \\
\hline $\begin{array}{c}\text { Preoperative Macula- } \\
\text { off (weeks) }\end{array}$ & $\begin{array}{c}0.03 \\
(p=0.78)\end{array}$ & 1 & & & & & & \\
\hline $\begin{array}{c}\text { Preoperative BCVA } \\
\text { (logMAR) }\end{array}$ & $\begin{array}{c}-0.07 \\
(p=0.47) \\
\end{array}$ & $0.04(p=0.68)$ & 1 & & & & & \\
\hline BCVA Before ERM and & -0.18 & $-0.16(p=0.1)$ & -0.10 & 1 & & & & \\
\hline
\end{tabular}




\begin{tabular}{|c|c|c|c|c|c|c|c|}
\hline ILM removal (logMAR) & $(p=0.07)$ & & $(p=0.33)$ & & & \\
\hline $\begin{array}{c}\text { ERM Detection } \\
\text { (weeks) }\end{array}$ & $\begin{array}{c}-0.18 \\
(p=0.21)\end{array}$ & $0.03(p=0.83)$ & $\begin{array}{c}-0.29 \\
(p=0.04)\end{array}$ & $\begin{array}{c}-0.16 \\
(p=0.26)\end{array}$ & 1 & & \\
\hline $\begin{array}{c}\text { Final Postoperative } \\
\text { BCVA (logMAR) }\end{array}$ & $\begin{array}{c}-0.04 \\
(p=0.72)\end{array}$ & $\begin{array}{c}-0.05 \\
(p=0.62)\end{array}$ & $0.10(p=0.3)$ & $0.78(p=0)$ & $\begin{array}{c}0.04 \\
(p=0.76)\end{array}$ & 1 & 0.02 \\
\hline CSFT (microns) & $\begin{array}{c}0.15 \\
(p=0.28)\end{array}$ & $0.32(p=0.02)$ & $0.02(p=0.89)$ & $\begin{array}{c}-0.14 \\
(p=0.32)\end{array}$ & $\begin{array}{c}0.02 \\
(p=0.89)\end{array}$ & $0.02(p=0.88)$ & 1 \\
\hline $\begin{array}{c}\text { Follow-up period } \\
\text { (months) }\end{array}$ & $\begin{array}{c}-0.14 \\
(p=0.18)\end{array}$ & $\begin{array}{c}-0.08 \\
(p=0.42)\end{array}$ & $0.09(p=0.36)$ & $\begin{array}{c}0.12 \\
(p=0.42)\end{array}$ & $-0.05(p=0.61)$ & $\begin{array}{c}-0.08 \\
(p=0.6)\end{array}$ & 1 \\
\hline
\end{tabular}

Wilcoxon rank sum test. The $p$-values in parenthesis ( $p)$; significant correlations $(p<0.05)$ are in bold text. BCVA: best corrected visual acuity; ERM: epiretinal membrane; ILM: internal limiting membrane; CSFT: central subfoveal thickness. Spearman Rank Test nonpeeling sample $=55$ eyes. Peeling sample $=50$ eyes.

Table S13: A) Preoperative, B) postoperative, and C) final BCVA in the Vitrectomy group (peeling and nonpeeling groups)

\section{A. preoperative BCVA (logMAR) Mann-Whitney $U$ tests results}

\begin{tabular}{|c|c|c|}
\hline & & \\
\hline Object & $\mathrm{U}$ & $\mathrm{p}$-value \\
\hline Age & 5565 & 0.001 \\
\hline Macula-off (weeks) & 5341 & 0.001 \\
\hline Follow-up period (days) & 5565 & 0.001 \\
\hline BCVA before ERM-ILM removal (logMAR) & 238 & 0.001 \\
\hline ERM detection (weeks) & 1326 & 0.001 \\
\hline Cinal postoperative BCVA (logMAR) & 0 & 0.001 \\
\hline CSFT (microns) & 1540 & 0.001 \\
\hline Follow-up period (months) & 4950 & 0.001 \\
\hline
\end{tabular}

B. postoperative BCVA (logMAR) Mann-Whitney $U$ tests results

\begin{tabular}{|c|c|c|}
\hline Object & & \\
\hline Age & $\mathrm{U}$ & $\mathrm{p}$-value \\
\hline Macula-off (weeks) & 5565 & 0.001 \\
\hline Preoperative BCVA (logMAR) & 5556 & 0.001 \\
\hline Follow-up period (days) & 4712 & 0.001 \\
\hline ERM detection (weeks) & 5565 & 0.001 \\
\hline Final postoperative BCVA (logMAR) & 1326 & 0.001 \\
\hline CSFT (microns) & 101.5 & 0.001 \\
\hline Follow-up period (months) & 1540 & 0.001 \\
\hline C. final BCVA after ERM proliferation removal (logMAR) & 5049 & 0.001 \\
\hline Object & & \\
\hline Age & & \\
\hline Macula-off (weeks) & $\mathrm{U}$ & $\mathrm{p}$-value \\
\hline Preoperative BCVA (logMAR) & 5565 & 0.001 \\
\hline Follow-up period (days) & 5565 & 0.001 \\
\hline ERM detection (weeks) & 5460 & 0.001 \\
\hline CSFT (microns) & 5565 & 0.001 \\
\hline Follow-up period (months) & 1074.5 & 0.001 \\
\hline BCVA before ERM-ILM removal (logMAR) & 1326 & 0.001 \\
\hline (O.05) are bold text BCVA best & 1540 & 0.001 \\
\hline
\end{tabular}

The statistically significant variables $(p<0.05)$ are in bold text. BCVA: best corrected visual acuity; ERM: epiretinal membrane; ILM: internal limiting membrane; CSFT: central subfoveal thickness 
Table S14: A) preoperative, B) postoperative, and C) final BCVA in the Vitrectomy group (peeling and nonpeeling groups)

\section{A. preoperative BCVA (logMAR) Kruskal-Wallis tests results}

\begin{tabular}{|c|c|c|c|}
\hline Object & Kruskal-Wallis $\mathrm{x}^{2}$. & df & p-value \\
\hline Male & 0.458 & 1 & 0.499 \\
\hline Eye & 1.878 & 1 & 0.171 \\
\hline Preoperative Lens Status & 1.640 & 1 & 0.200 \\
\hline Preoperative ERM proliferations & 0.760 & 1 & 0.383 \\
\hline First Surgery & 1.055 & 2 & 0.590 \\
\hline BCVA Before ERM-ILM removal & 9.412 & 12 & 0.667 \\
\hline Recurrent RRD & 0.208 & 1 & 0.649 \\
\hline Additional surgery & 1.360 & 4 & 0.851 \\
\hline Postoperative ERMs & 0.038 & 1 & 0.846 \\
\hline ERM 2nd surgery & 3.135 & 3 & 0.371 \\
\hline Final Postoperative BCVA & 11.718 & 10 & 0.304 \\
\hline Foveal contour abnormalities & 0.385 & 1 & 0.535 \\
\hline Ellipsoid disruption & 4.175 & 1 & 0.041 \\
\hline DONFL defects & 1.402 & 1 & 0.236 \\
\hline ELM line alterations & 0.144 & 1 & 0.704 \\
\hline mfERG alterations & 0.109 & 1 & 0.741 \\
\hline Microperimetry alterations & 1.623 & 1 & 0.203 \\
\hline \multicolumn{4}{|c|}{ B. postoperative BCVA (logMAR) Kruskal-Wallis tests results } \\
\hline Object & Kruskal-Wallis $x^{2}$. & df & $p$-value \\
\hline Male & 0.355 & 1 & 0.552 \\
\hline Eye & 0.001 & 1 & 0.979 \\
\hline Preoperative Lens Status & 6.083 & 1 & 0.014 \\
\hline Preoperative BCVA & 12.845 & 8 & 0.117 \\
\hline Preoperative ERM proliferations & 50.177 & 1 & 0.001 \\
\hline First surgery & 47.013 & 2 & 0.000 \\
\hline Recurrent RRD & 11.364 & 1 & 0.001 \\
\hline Additional surgery & 12.324 & 4 & 0.015 \\
\hline Postoperative ERM proliferations & 68.366 & 1 & 0.001 \\
\hline ERM 2nd surgery & 5.469 & 3 & 0.141 \\
\hline Foveal Contour abnormalities & 10.021 & 1 & 0.002 \\
\hline Ellipsoid disruption & 1.091 & 1 & 0.296 \\
\hline DONFL defect & 19.206 & 1 & 0.001 \\
\hline ELM line alterations & 0.746 & 1 & 0.388 \\
\hline mfERG alterations & 31.253 & 1 & 0.001 \\
\hline Microperimetry alterations & 19.749 & 1 & 0.001 \\
\hline \multicolumn{4}{|c|}{ C. final BCVA after ERM proliferation removal (logMAR) } \\
\hline Object & Kruskal-Wallis $x^{2}$ & $d f$ & $p$-value \\
\hline Male & 1.561 & 1 & 0.211 \\
\hline Eye & 0.121 & 1 & 0.728 \\
\hline Preoperative Lens Status & 1.855 & 1 & 0.173 \\
\hline Preoperative ERM proliferations & 33.337 & 1 & 0.001 \\
\hline First surgery & 13.877 & 2 & 0.001 \\
\hline
\end{tabular}




\begin{tabular}{|c|c|c|c|}
\hline Recurrent RRD & 9.223 & 1 & 0.002 \\
\hline Additional surgery & 10.697 & 4 & 0.030 \\
\hline Postoperative ERM proliferations & 38.068 & 1 & 0.001 \\
\hline ERM 2nd surgery & 1.113 & 3 & 0.774 \\
\hline Foveal contour abnormalities & 6.168 & 1 & 0.013 \\
\hline Ellipsoid disruption & 0.894 & 1 & 0.344 \\
\hline DONFL defect & 16.777 & 1 & 0.001 \\
\hline ELM line alterations & 0.375 & 1 & 0.540 \\
\hline mfERG alterations & 16.522 & 1 & 0.001 \\
\hline Microperimetry alterations & 13.150 & 1 & 0.001 \\
\hline
\end{tabular}

The statistically significant variables $(p<0.05)$ are in bold text. df: degrees of freedom: BCVA: best corrected visual acuity; ERM: epiretinal membrane; ILM: internal limiting membrane; RRD: rhegmatogenous retinal detachment; DONFL: diffuse optic nerve fiber layer; ELM: external limiting membrane: mfERG: multifocal electroretinography

Table S15: Generalized Linear Model results A) "Preoperative, B) Postoperative, and C) final BCVA in the Vitrectomy group (peeling and nonpeeling groups)

A. preoperative BCVA (logMAR) GLM results

\begin{tabular}{|c|c|c|c|c|}
\hline & & & & \\
\hline & Estimate & SE & t value & $p$ \\
\hline (Intercept) & 1.055 & 0.026 & 40.60 & 0.001 \\
\hline
\end{tabular}

\section{B. postoperative BCVA (logMAR) GLM results}

\begin{tabular}{|c|c|c|c|c|}
\hline & Estimate & SE & t value & $\mathrm{p}$ \\
\hline (Intercept) & 0.515 & 0.067 & 7.72 & 0.001 \\
\hline Postoperative ERM proliferations & 0.448 & 0.050 & 8.97 & 0.001 \\
\hline First surgery - ONLY VITRECTOMY & -0.235 & 0.055 & -4.30 & 0.001 \\
\hline First surgery - VIT ERM and ILM REMOVAL & 0.034 & 0.090 & 0.38 & 0.704 \\
\hline Macula-off (weeks) & -0.019 & 0.008 & -2.50 & 0.014 \\
\hline Recurrent RRD & 0.118 & 0.061 & 1.94 & 0.055 \\
\hline \multicolumn{5}{|c|}{ C. final BCVA after ERM proliferation removal (logMAR) GLM results } \\
\hline & Estimate & SE & t value & $\mathrm{p}$ \\
\hline (Intercept) & -0.213 & 0.070 & -3.05 & 0.003 \\
\hline BCVA Before ERM-ILM removal (logMAR) & 0.552 & 0.046 & 11.98 & 0.001 \\
\hline First Surgery - ONLY VITRECTOMY & 0.201 & 0.038 & 5.22 & 0.001 \\
\hline First Surgery - VIT ERM and ILM REMOVAL & 0.275 & 0.051 & 5.42 & 0.001 \\
\hline Preoperative BCVA (logMAR) & 0.106 & 0.046 & 2.29 & 0.024 \\
\hline Gender - Male & 0.052 & 0.026 & 2.03 & 0.045 \\
\hline
\end{tabular}

The statistically significant variables $(p<0.05)$ are in bold text. BCVA: best corrected visual acuity; GLM: generalized linear models; SE: standard error; ERM: epiretinal membrane; VIT: vitrectomy; ILM: internal limiting membrane; mfERG: multifocal electroretinography; $R R D$ : rhegmatogenous retinal detachment 


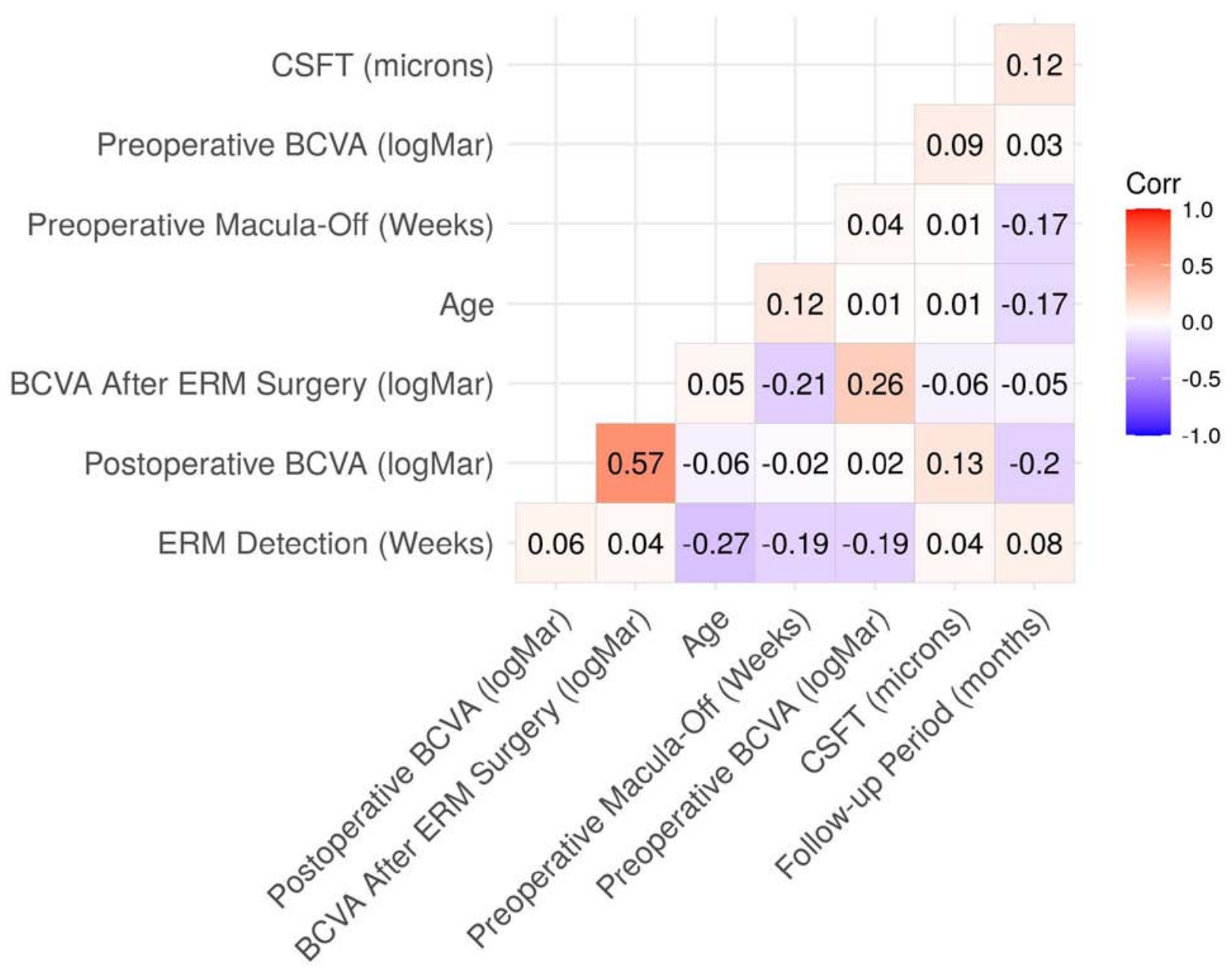

Additional Figure S1: The Spearman's rank correlation coefficient test showed that there was a moderate to strong positive correlation ( $r h o=0.57, p<0.01$ ) of the postoperative BCVA in logMAR units with the BCVA after ERM surgery. In addition, there was a weak negative correlation (rho $=-0.2, p<0.05$ ) between postoperative BCVA in logMAR units and follow-up period in months. 


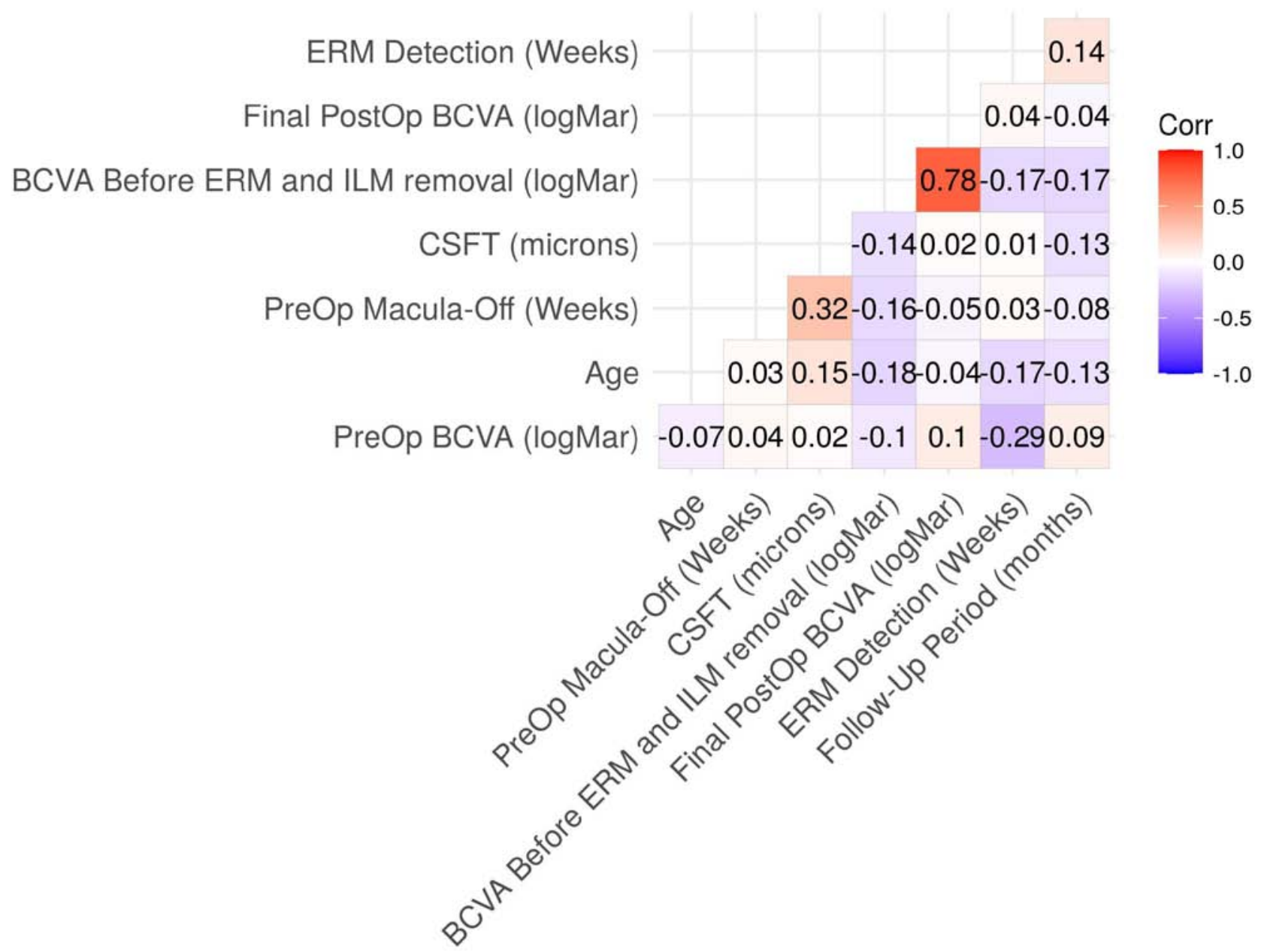

Additional Figure S2: The Spearman's rank correlation coefficient test showed a weak positive correlation (rho $=0.32$, $p<0.05)$ between the preoperative period with the macula-off in weeks and the CSFT findings in microns; it also showed a weak negative correlation ( $r h o=-0.29, p<0.05$ ) between the preoperative BCVA in logMAR units and ERM detection in weeks. 


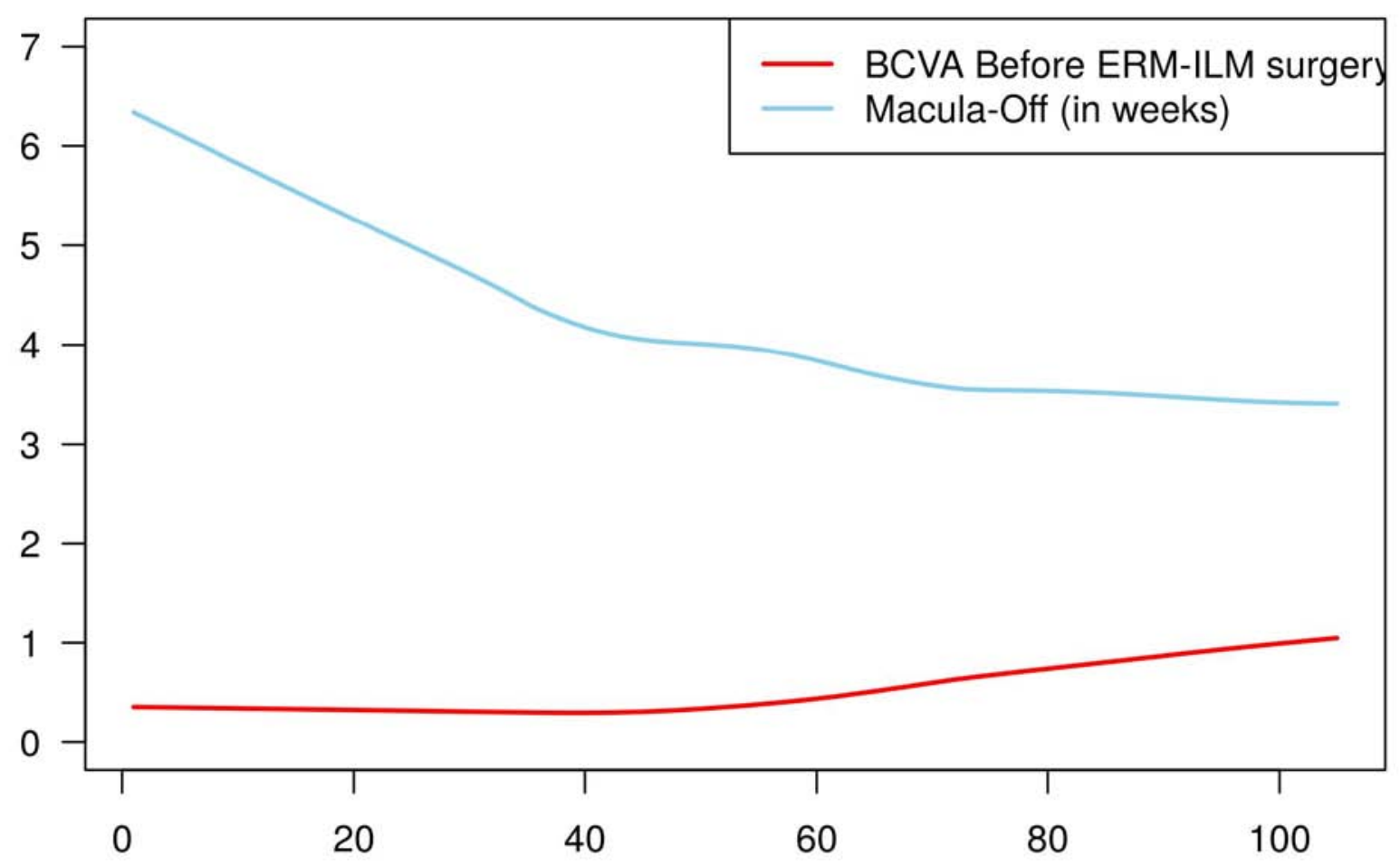

Additional Figure S3: Postoperative BCVA was significantly negatively associated when only vitrectomy (non-peeling group) was performed in the first surgery variable (coefficient $=-0.23, p<0.01$ ); and significantly negatively associated with the variable preoperative period of macula-off in weeks (coefficient $=-0.02, p<0.05$ ) is shown.

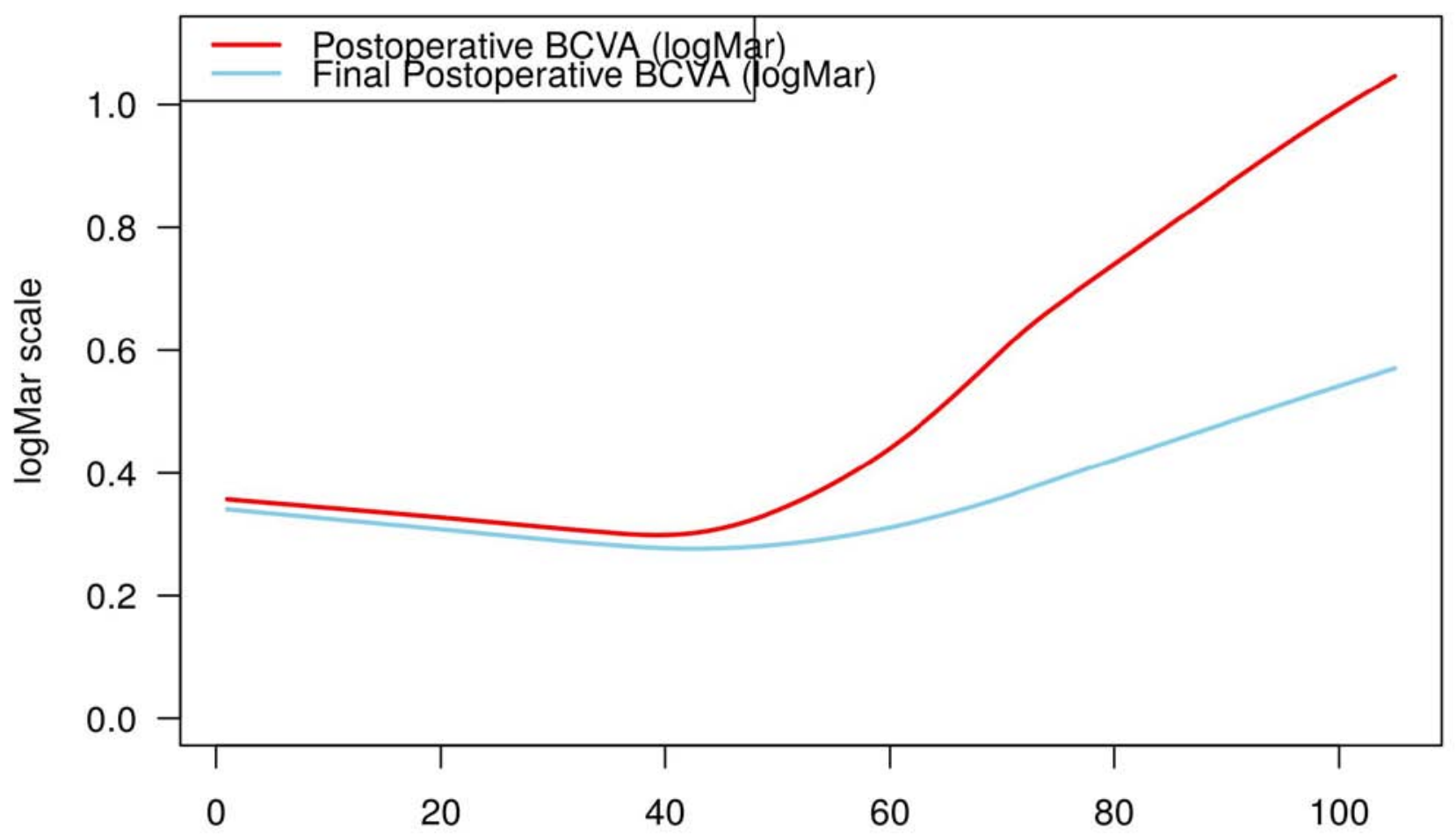

Additional Figure S4: The GLM for the final BCVA in log MAR units after ERM proliferation removal showed that it was significantly positively associated ( $p>0.01$ ) with the postoperative 


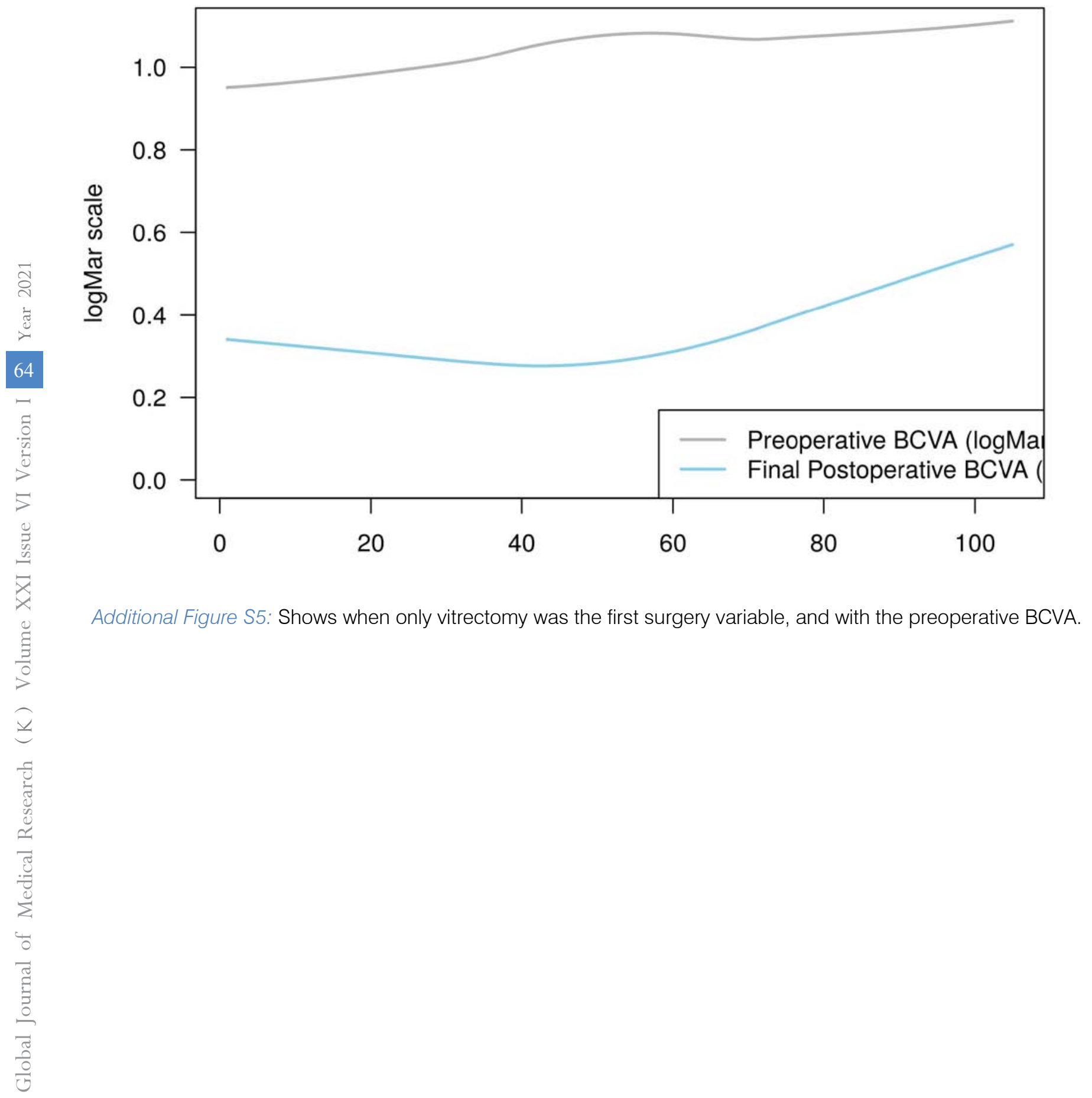

